\title{
Heat transfer across a fractal surface
}

\author{
Michael Frank $^{1 *}$, Michail Papanikolaou ${ }^{2}$, Dimitris Drikakis ${ }^{3}$, Konstantinos Salonitis $^{2}$ \\ ${ }^{1}$ University of Strathclyde, Glasgow, G1 1XW, United Kingdom \\ ${ }^{2}$ Manufacturing Theme, Cranfield University, Cranfield, MK43 OAL, United Kingdom \\ ${ }^{3}$ University of Nicosia, Nicosia, Cyprus \\ *Corresponding Author: michael.frank@strath.ac.uk
}

\begin{abstract}
The effects of surface irregularities and imperfections on the thermal resistance at a solidliquid interface have been investigated using molecular dynamics. The molecular model comprises liquid argon confined between silver walls. The surface roughness was designed using fractal theory, introducing stochastic patterns of multiple scales that resemble realistic surface geometries. In agreement with most previous studies, we find that increasing the strength of the solid-liquid interactions, monotonically reduces the thermal resistance across smooth interfaces. Yet, the behaviour of the thermal resistance across rough surfaces is more complex. Following the initially anticipated decrease, the thermal resistance starts to increase once the strength of solid-liquid interaction increases past a threshold. We attribute the above behaviour to two competing phenomena, namely the area of the solid-liquid interface, and the introduction of vibrational anharmonicities and localisation of phonons resulting from the surface roughness. Finally, we demonstrate that, for the same fractal dimension and depth of surface roughness, different surfaces practically have the same thermal resistance, solid-liquid radial distribution function and liquid density profiles. We conclude that the above fractal parameters are useful in deriving reduced models for properties related to the surface geometry.
\end{abstract}

\section{Introduction}

The development of complex systems such as high performance computing and aerospace electronic equipment, have established thermal management as a key design consideration. Liquid cooling is an effective way to release excess heat from the systems. As the characteristic dimensions of the system decrease, the surface-to-volume ratio increases, and therefore the efficiency of liquid cooling should, in theory, increase as well. Yet at nanoscale dimensions, a common bottleneck is the thermal resistance at the solid-liquid interface, which impedes the transfer of heat into the liquid.

The Interfacial Thermal Resistance (ITR) between two materials can be described asymptotically by two models: the Acoustic Mismatch Model (AMM) [1] and the Diffuse Mismatch Model (DMM) [2]. The AMM treats phonons as planar waves with wavelengths significantly greater than the atomic spacing. Waves incident on the interface will either be transmitted (refraction) or reflected, depending on a transmission probability that is itself a function of the acoustic impedances of the two materials. A major assumption of AMM is that all reflections are specular. Interfaces exhibiting many scattering events-such as systems at high temperatures or surfaces that contain surface roughness-are inaccurately captured by the AMM. The DMM assumes that all incident phonons that are not transmitted, are reflected diffusively. Both theories are based on strong assumptions that do not generally hold. 
The AMM and DMM also ignore significant molecular phenomena. Molecular interactions between the solid and liquid regulate the momentum transfer between the two materials. Furthermore, the liquid in the vicinity of a solid surface has different vibrational properties compared to the same liquid in its bulk form [3], which depend on the wetting properties of the solid [4]. Increasing the strength of the solid-liquid interactions exponentially decreases the ITR $[5,6,7,8]$, a fact that the AMM and DMM completely dismiss. The molecular mass of the solid wall also affects the thermal resistance, with an increase in molecular mass resulting in an increase of ITR [9]. The density of liquids in the vicinity of a smooth solid surface fluctuates due to the formation of structured liquid layers parallel to the surface, which is in turn a result of the solid-liquid interactions. A MD study found that the liquid density of the first such layer adjacent to a solid was strongly correlated to the thermal resistance [10]. A computational investigation has shown that the crystallographic orientation of silicon affects the thermal resistance at the silicon/water interface despite the fact that both orientations resulted in the same wetting properties for the solid.

Many of the computational studies on ITR consider atomically flawless solid surfaces, an idealistic simplification considering that most realistic surfaces are inherently rough. Surface roughness exposes more surface area to the liquid, theoretically facilitating energy transfer across the interface. Additionally, surface roughness affects the solid's wetting properties [11, $12,13,14]$; its effect on the wettability of the solid depends on the width and depth of the protrusions of the wall, as well as on the type of liquid at the interface. Surface roughness can also increase the number of phonon scattering events [2] which could potentially have an impact on ITR.

Previous MD studies have investigated the effect of roughness on the ITR. Periodic, rectangular patterns on a solid surface reduce the thermal resistance, with the reduction being accentuated when increasing the depth and width of the rectangular protrusions [15]. A two-dimensional molecular model was proposed based on fractal Cantor sets to create rectangular protrusions of different frequency and height [16]. MD simulations using the above model showed that an increase of the average height of the protrusions and the level of surface irregularity reduces the ITR. Furthermore, the thermal resistance between liquid water and self-assembled monolayers-a combination of $\mathrm{CF} 3$ and $\mathrm{OH}$ groups-is also decreased on rough surfaces due to the larger surface area exposed to water [17]. But when normalised by the area of the rough surface, the intrinsic thermal resistance showed different, and subtler changes: the thermal resistance increased for the more irregular surfaces but decreased when a smooth sinusoidal surface was considered. A more recent study investigated the interfacial thermal conductance at the wall, considering rectangular protrusions of different depth and width, as well as different values for the strength of the solid-liquid interactions [18]. The conclusion of the paper was that, at hydrophobic surfaces, larger groves resulted in lower thermal conductance than smaller ones, since the liquid's surface tension would prevent it from filling up the grooves, resulting in a smaller effective area for heat transfer, i.e. the Cassie regime [19]. At hydrophilic surfaces, i.e. the Wenzel regime [12], larger grooves resulted in greater conductance, and roughness seemed to increase conductance, relative to the smooth surface.

MD models previously used to study the effects of roughness on thermal resistance are either two-dimensional models, or model surface roughness as simple oscillations of a certain wavenumber and amplitude. The authors have shown that a more realistic, three- 
dimensional fractal model predicts different thermodynamic properties [20] and flow dynamics [21] than 2D models.

Using fractal theory to design roughness that mimics the multiple scales of realistic surfaces and performing MD simulations for such surfaces can shed light on interfacial phenomena and parameters that affect the ITR; this is the overall aim of this study. The multivariate Weierstrass-Mandelbrot (W-M) fractal function has been employed for generating two symmetric rough walls. Non-equilibrium molecular dynamics (NEMD) has been employed to study ITR for different strength values of the solid-liquid interaction.

\section{Methodology}

The model consists of liquid argon between two silver walls. Rough wall geometries have been generated using the multivariate $\mathrm{W}-\mathrm{M}$ function [22]:

$$
\begin{aligned}
& y(x, z)=C \sum_{m=1}^{M} \sum_{n=0}^{n_{\max }} \gamma^{\left(D_{s}-3\right)}\left\{\cos \Phi_{m, n}-\cos \left[\frac { 2 \pi \gamma ^ { n } \sqrt { ( x ^ { 2 } + z ^ { 2 } ) } } { L _ { M A X } } \cdot \operatorname { c o s } \left(\tan ^{-1}\left(\frac{z}{x}\right)-\right.\right.\right. \\
& \left.\left.\left.\frac{\pi m}{M}\right)+\Phi_{m, n}\right]\right\} \text {. }
\end{aligned}
$$

W-M embeds $M$ surfaces and each one of them is a superposition of $\mathrm{n}_{\max }$ different frequencies. The frequencies on each surface are indexed by the integers $n$ and $m$ respectively and are offset by a random phase $\Phi_{m, n}$. The parameter $\gamma$ is the frequency density and $L_{M A X}$ is the size of the sample; in the present study $L_{M A X}=L_{x}=L_{z}$. The parameter $D_{s}$ is the fractal dimension, an indication of the fractal's ability to fill up space. For a threedimensional fractal, $2<D_{s}<3$. Finally, $C$ is a scaling factor determining the average amplitude of the waves:

$$
C=L_{M A X}\left(\frac{G}{L_{M A X}}\right)^{D_{S}-2}\left(\frac{\ln \gamma}{M}\right)^{1 / 2},
$$

where $G$ is the roughness parameter that can be adjusted to obtain different depths of roughness.

In the theoretical limit, a perfect fractal corresponds to an infinite number of frequencies, i.e. $n_{\max } \rightarrow \infty$. However, a more practical value can be selected by using

$$
n_{\max }=\left[\frac{\log \left(L_{\max } / L_{\min }\right)}{\log \gamma}\right]
$$

where $L_{\min }$ is the smallest wavelength in the system. Equation (3) ensures that the wavelengths span from $L_{\min }$ to $L_{\max }$. In general, the frequency density can take any value greater than one and in this paper $\gamma=1.5$, which does not result in repeated wavelengths; another commonly used value is $\gamma=5$.

The dimensions of the simulation box in the $x, z$ and $y$ directions were set equal to $L x=L z=$ $7.4 \mathrm{~nm}$, and $L y=14.4 \mathrm{~nm}$, respectively (Figure 1). Each geometry starts as a single smooth solid block, corresponding to the lower wall. This initial solid block consists of 22 layers of 
particles located perpendicular to the $y$ direction. We then use the middle of this wall, i.e. the $11^{\text {th }}$ layer, as a reference point, henceforth refer to as the centreline. Starting from this centreline we compute the $\mathrm{W}-\mathrm{M}$ function for different values of $\mathrm{G}$, and delete all atoms that are located above this function. For the smooth wall we delete all layers above the centreline, i.e. the smooth wall consists of 11 layers. We obtain the upper wall through reflection.

The fractal dimension considered here is $D_{s}=2.5$ corresponding to a highly irregular topography. For this study we have considered three different roughness amplitudes, $G=0$, $G=0.4$ and $G=0.8$, where $G=0$ corresponds to a smooth, atomically flawless wall and $G=$ 0.8 corresponds to a surface with large fluctuations (Figure 1 ). To quantify the uncertainty related to the stochastic nature of the fractal model, we generated three different geometries for each non-zero value of $\mathrm{G}$ (i.e. $\mathrm{G}=0.4$ and $\mathrm{G}=0.8$ ) by adjusting the random phase $\Phi_{m, n}$. Thus we consider seven geometries in total: one for $G=0$, three for $G=0.4$, and three for $G=0.8$.

All simulations were carried out using the open-source MD simulator LAMMPS [23]. The wallfluid and fluid-fluid interactions were modelled using the 12-6 Lennard-Jones (LJ) potential:

$$
v_{i j}^{L J}=4 \varepsilon\left[\left(\frac{\sigma}{r_{i j}}\right)^{12}-\left(\frac{\sigma}{r_{i j}}\right)^{6}\right],
$$

where $\varepsilon$ is the depth of the potential well and quantifies the strength of the interaction, $\sigma$ is the van der Waals radius and $r_{i j}$ is the distance between particles $i$ and $j$. For the fluid-fluid interactions we used the parameters $\varepsilon_{f f}=0.0104 \mathrm{eV}$ and $\sigma_{f f}=3.405 \AA$. The strength of the solid-liquid interaction is a parameter of interest. Thus we have considered six different values: $\varepsilon_{w f}=0.002 \mathrm{eV}, \quad \varepsilon_{w f}=0.008 \mathrm{eV}, \varepsilon_{w f}=0.020 \mathrm{eV}, \varepsilon_{w f}=0.040 \mathrm{eV}, \varepsilon_{w f}=$ $0.060 \mathrm{eV}$, and $\varepsilon_{w f}=0.080 \mathrm{eV}$.

The wall atoms were modelled using the EAM potential:

$$
v_{i}^{E A M}=\sum_{j \neq 1} v\left(r_{i j}\right)+f\left(\rho\left(r_{i j}\right)\right),
$$

where $v_{i j}$ is a pair potential interaction, $\rho\left(r_{i j}\right)$ is the local electron density and $f(\rho)$ is an embedding function. The three parameters for Eq. 5 are read from a large file [24]; we used the file from the National Institute of Standards and Technology web site, at https://www.ctcms.nist.gov/potentials. The lattice constant was set equal to $4.086 \AA$ which corresponds to silver. 

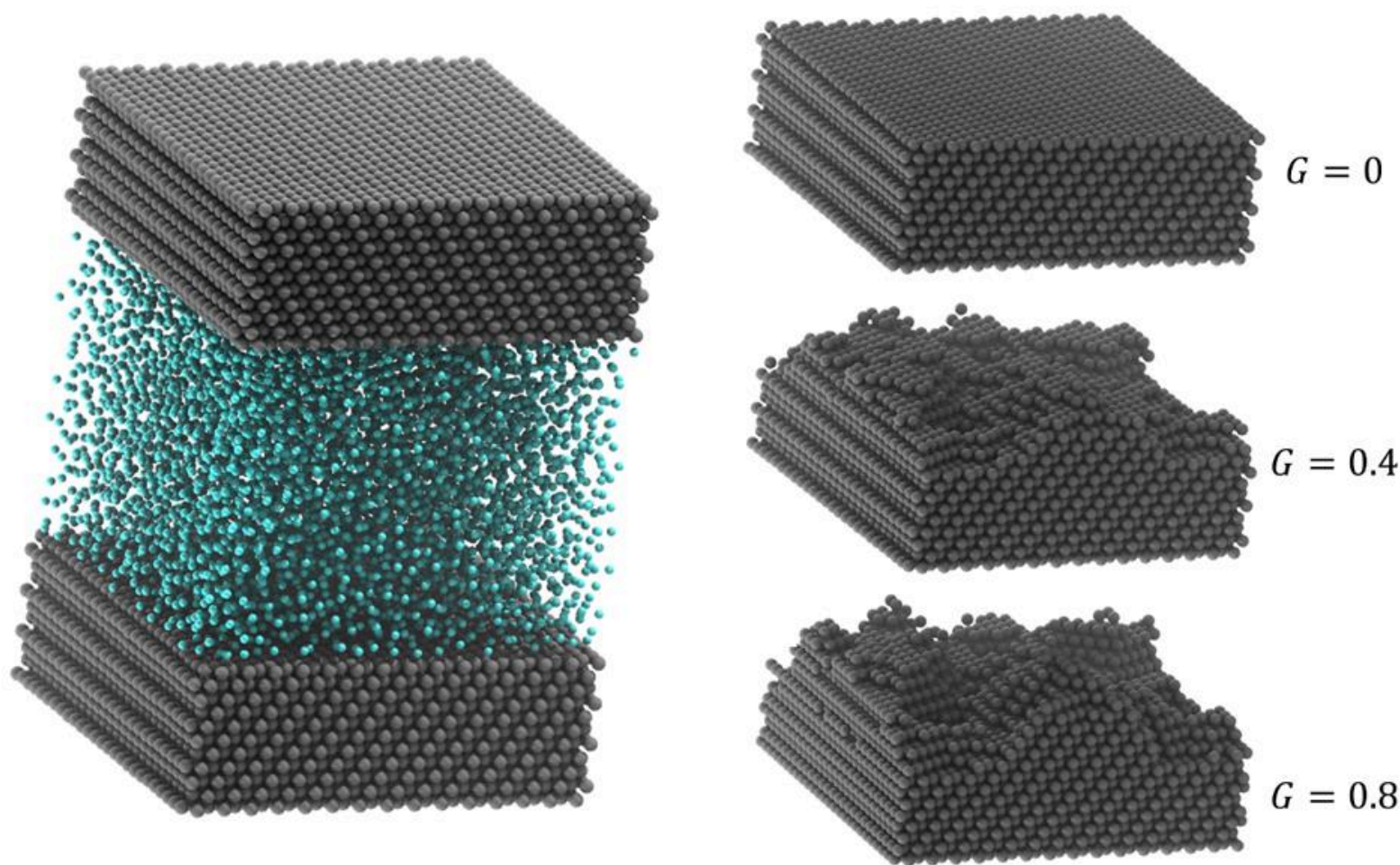

Figure 1: Left: MD model illustrating liquid argon (cyan) confined by two silver walls (grey). Right: Surfaces of different depth of roughness.

The cutoff distance is $7.495 \AA$ and the silver and argon masses were $m_{A g}=107.9 \mathrm{~g} / \mathrm{mol}$ and $m_{A r}=39.948 \mathrm{~g} / \mathrm{mol}$, respectively. Due to the complex nature of the wall geometry, the dynamic Voronoi tessellation on the position of the atoms was employed to calculate the volume of the channel. The number of liquid atoms varied between cases to retain a constant density of $1.4 \mathrm{~g} / \mathrm{cm}^{3}$.

To control the temperature of the system the Langevin thermostats were applied in the first four outmost layers of the silver walls, which were also attached onto their initial lattice sites by springs [25]. A temperature difference between the lower and the upper wall was induced by setting $T_{\text {low-wall }}=90 \mathrm{~K}$ and $T_{\text {high-wall }}=140 \mathrm{~K}$.

Time integration was performed using the Verlet algorithm, with a timestep equal to $5 \times 10^{-3} \mathrm{ps}$. An initialisation period of $10^{6}$ timesteps was used to ensure a linear temperature profile across the channel height, and a converged pressure. Following the initialisation phase, the system was sampled for a further $2 \times 10^{6}$ timesteps for the calculation of the thermal resistance.

To measure the temperature across the nanochannel the equipartition theorem was used:

$$
T=\frac{2}{3 N k_{b}} \cdot\left\langle E_{k i n}\right\rangle,
$$

where $\left\langle E_{k i n}\right\rangle$ is the total kinetic energy, $N$ the total number of atoms and $k_{b}=1.38$. $10^{-23} \mathrm{~m}^{2} \mathrm{kgs}^{-2} \mathrm{~K}^{-1}$ the Boltzmann constant. ITR can be estimated according to: 


$$
R_{k}=\frac{\Delta T}{q}=\frac{\Delta T}{Q / A}
$$

where $\Delta T=T_{\text {liquid }}-T_{\text {wall }}$ is the temperature jump at the interface and $q$ is the heat flux. The heat flux is calculated by the heat $Q$ generated by the Langevin thermostats divided by the surface area $A$ of the thermostat.

To calculate the temperature jump we used linear regression to compute continuous temperature profiles for the liquid and wall. We then calculated the temperature jump as the difference between the two profiles at the location of the centreline. As described earlier, this corresponds to the location of the surface of the smooth wall.

A final note, due to the irregular nature of the geometry under consideration, the local thermal conductance changes across the solid surface. The calculated thermal resistance corresponds to the inverse of the conductance averaged across the entire rough surface.

To investigate the liquid structure next to the solid wall, we calculate the liquid density profiles. Specifically, we used a scheme very similar to that proposed by Willard and Chandler [26]. In brief, the density profiles are calculated by measuring the minimum distance between each liquid atom and the wall. These distances are segregated into a histogram of bin size $0.05 \AA$. The number of atoms in each bin is then normalised by the product of the dimensions of the simulation box parallel to the mean orientation of the surface (i.e. $L_{x}, L_{z}$ ), and the bin size, i.e. $74 \times 74 \times 0.05 \AA=273.8 \AA^{3}$. A slight discrepancy between the method used here and that by Willard and Chandler [26] is that in their approach, they identified the interface using density iso-surfaces; subsequently the distances considered were those between the liquid atoms and these iso-surfaces. The complex structure of our geometries results in pretty discontinuous iso-surfaces $[20,21]$. Instead, we identified the interface as the solid atoms that are within a distance of $4 \AA$ from liquid atoms (the value of $4 \AA$ was obtained from the Radial Distribution Functions (RDF) and is the distance that includes the first peak). Also, Willard and Chandler distinguish between a mean and instantaneous interface; here the two coincide as the surface remains constant with time.

To better understand the underlying physical mechanisms behind the thermal resistance, we study the phonon behaviour in the solid walls. We do this by considering the Vibrational Density of States (VDOS) of the solid walls. This is calculated by Fourier transforming the velocity autocorrelation function of the wall atoms. Specifically, we sample the atomic velocities every 15 timesteps, for a total of 10500 timesteps. We then calculate the autocorrelation function of the velocities. The VDOS are obtained by calculating the spectra of the velocity autocorrelation function, which we obtain through the Welch method with a $50 \%$ overlapping window $[27,28]$. To reduce statistical noise, we averaged the VDOS over 10 different samples, i.e. simulations with the same macrostate but starting from a different microstate. We also normalise the VDOS by the number of solid atoms used for the calculation, as this differs between different cases.

Finally, to gain insight into the dynamic behaviour of the phonons, we consider the Heat Flux Autocorrelation Function (HFACF) within the solid. This is calculated as the autocorrelation function of the microscopic heat flux, the latter of which is defined as: 


$$
\boldsymbol{J}=\frac{1}{V}\left(\sum_{i} E_{i} \boldsymbol{v}_{i}+\frac{1}{2} \sum_{i<j}\left(\boldsymbol{f}_{i j} \cdot \boldsymbol{v}_{j}\right) r_{i j}\right)
$$

where the subscripts $i$ and $j$ denote two arbitrary atoms; $E_{i}$ and $\boldsymbol{v}_{i}$ are the total energy and velocity, respectively, of atom $i$; and $\boldsymbol{f}_{i j}$ and $r_{i j}$ are the interatomic force, and interatomic distance, respectively, between atoms $i$ and $j$. For the calculation of the HFACF we used a correlation length of $10^{4}$. Furthermore, to minimise statistical noise we averaged the HFACF over 30 samples for each case. Finally, Eq. 8 only considers the solid atoms at the cold wall, and excludes atoms in the four initial layers that are attached by springs, i.e. the indices $i$ and $j$ only run through these atoms.

The integral of the HFACF is proportional to the thermal conductivity, as described by the Green-Kubo relations. Note that the correlation length of $10^{4}$ timesteps is not long enough for the HFACF to converge to zero, as required by the Green-Kubo. In this study, however, we are only interested in the qualitative differences of the function between the different cases. To that end, our choice of correlation length provides the information that we require, while managing computational resources.

\section{Results}

For the majority of this study we focus on the cold wall as the behaviour of the thermal resistance at the hot and cold wall is qualitatively the same. Some minor quantitative differences are discussed at the end of this section.

The ITR distributions for different depths of surface roughness and strengths of the solidliquid interactions are shown in Figure 2a. For smooth surfaces, the ITR decreases monotonically with increasing strength of solid-liquid interaction, which agrees with most published results $[5,6,7,8]$. The above behaviour is related to better coupling between the two materials. Thus for very low values of $\varepsilon_{w f}$, the solid and liquid atoms practically vibrate independently of each other, while increasing $\varepsilon_{w f}$ increases the probability of phonon transmission across the interface.

In the presence of surface roughness, increasing $\varepsilon_{w f}$ initially results in a similar decay in ITR. As the solid-liquid interaction increases further the ITR starts to increase slightly (Figure 2a). Furthermore, the effect of surface roughness seems to be more prominent at lower values of $\varepsilon_{w f}$, where increasing the depth of surface roughness results in a decrease in thermal resistance(Figure $2 \mathrm{~b}$ ). For higher values of $\varepsilon_{w f}$, introducing and increasing the depth of surface roughness results in very little change and for $\varepsilon_{w f}=0.06 \mathrm{eV}$ and $\varepsilon_{w f}=0.08 \mathrm{eV}$, we even observe a slight increase in the thermal resistance. This finding is partly in disagreement with previous studies that usually show a strictly decreasing trend of the thermal resistance with roughness $[15,16,17]$.

For $\mathrm{G}>0$, the error bars in Figure 2 show the standard deviation of the thermal resistance for the three different geometries that share the same fractal parameters, $\mathrm{G}$ and $D_{s}$, for each case. For $\mathrm{G}=0$, where there is only one possible geometry, the error bars correspond to the standard deviation of the thermal resistance for three cases of identical macrostates, starting, however, from a different microstate. 
The atomic configuration of the randomly generated geometries is significantly different from each other. Yet we observe only small deviations for each case (i.e. for each combination of $\varepsilon_{w f}$ and $\left.\mathrm{G}\right)$. Furthermore, the deviation arising from the different geometries for $\mathrm{G}=0.4$ and $\mathrm{G}=0.8$ are of the same order as the statistical noise observed at $\mathrm{G}=0$. The constancy of the ITR for different geometries that share the same fractal parameters, suggests that fractal theory can be used toward a more universal characterisation of topography-related properties. Specifically, the fractal dimension and average depth of surface roughness (Eqs. 1 and 2), seem to be sufficient to capture the effect of surface geometry on the thermal resistance without explicit consideration of the molecular details. This finding is in agreement with previous studies on nanoscale grinding [29].

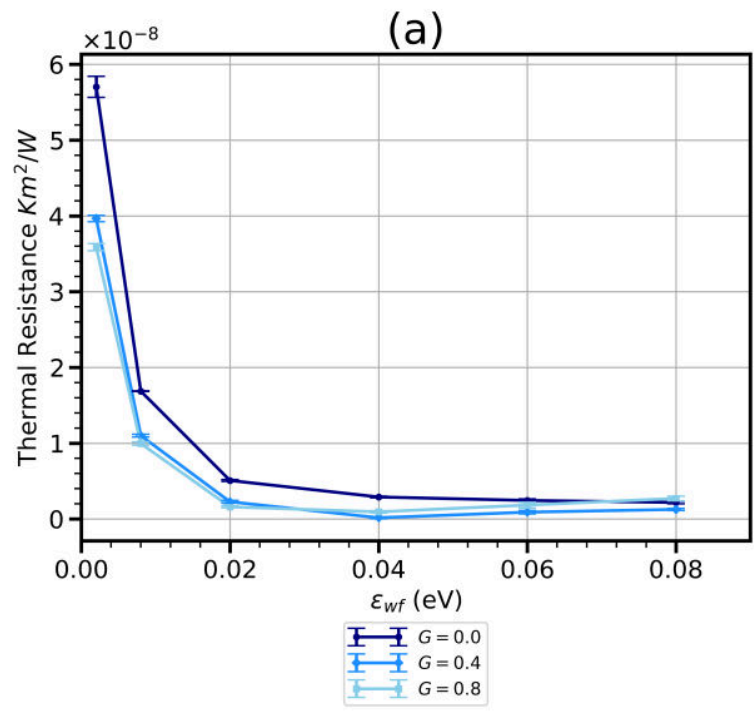

(b)

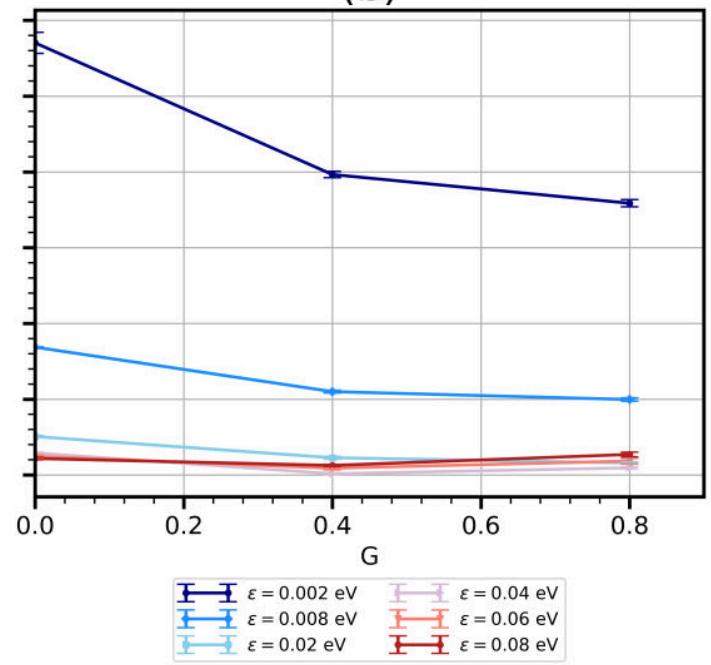

Figure 2 Thermal resistance as a function of (a) the strength of the solid-liquid interactions and (b) the depth of surface roughness

The decrease of the thermal resistance with surface roughness is, at least partly, due to the increase in interfacial area. Surface roughness, however, also affects the wetting properties of the solid, subsequently influencing the thermal resistance. For the same strength of solidliquid interactions, the RDF of the liquid in the vicinity of the solid atoms increases with the introduction of surface roughness, suggesting better wetting properties (Figure 3a).

Figure $3 \mathrm{~b}$ presents the liquid density profiles, obtained using the method proposed by Willard and Chandler [26] (see methodology). For cases with smooth walls, the density profiles show the usual sharp and well-separated peaks, indicative of the layered nature of the liquid in the vicinity of the wall. In the presence of roughness, the first peak consistently has a greater amplitude compared to the equivalent smooth case. Yet past the first peak, the density profiles gradually decrease and converge to their bulk value, showing only minor and smooth fluctuations.

We expect that the fractal dimension of the rough wall will have an effect on the liquid density. Previous studies have shown that the RDF of a solvent around a solute depends on the size of the solute [30]. Smaller solutes result in better wetting properties dueto the intermolecular forces between the solvent molecules. As the size of the solute increases, these forces weaken, thus resulting in a less wettable surface. Extrapolating from their results, we believe that a different fractal dimension, leading to less irregular geometries will result 
in different wetting properties and RDFs. The effect of fractal dimension on the wetting properties of a surface will be investigated in a future study.

(a)

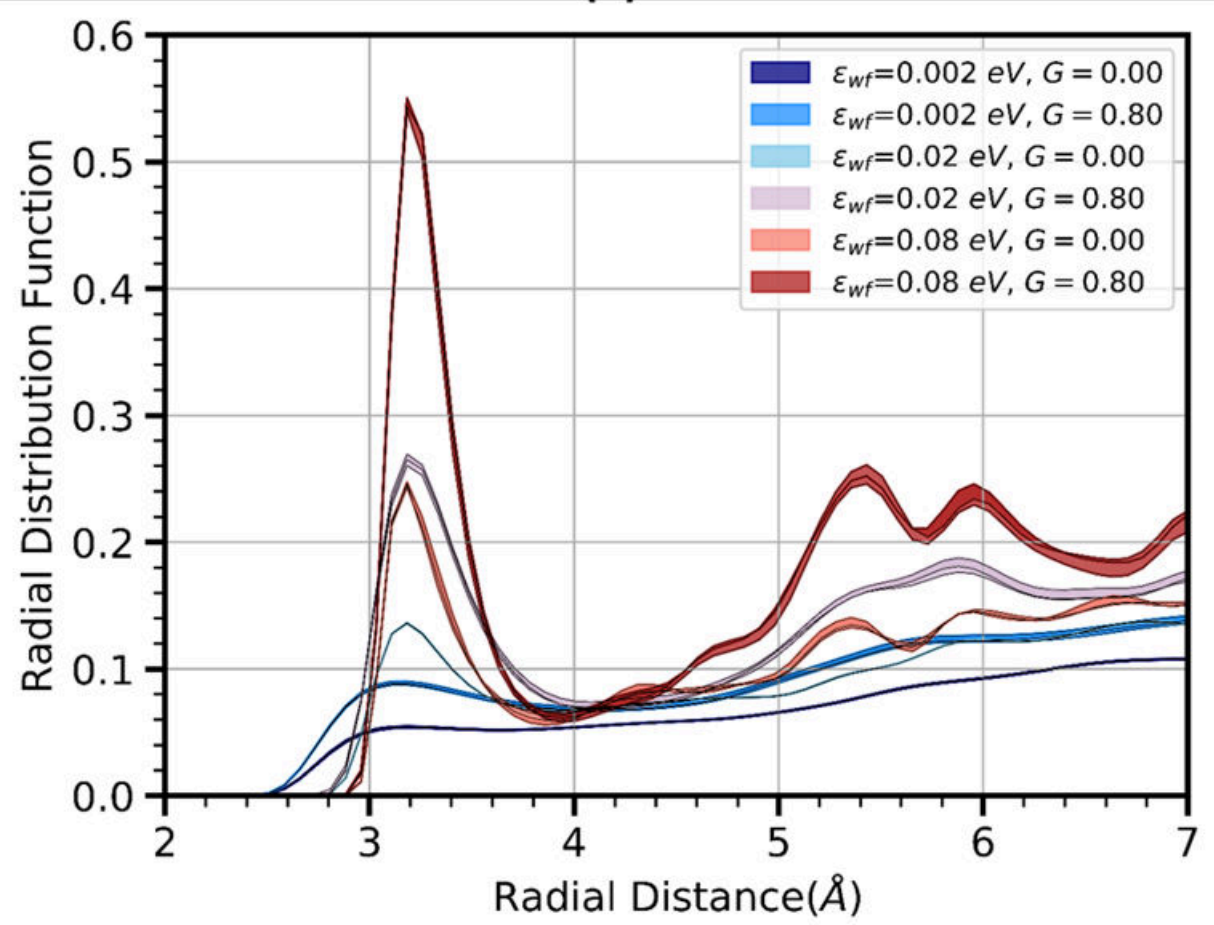

(b)

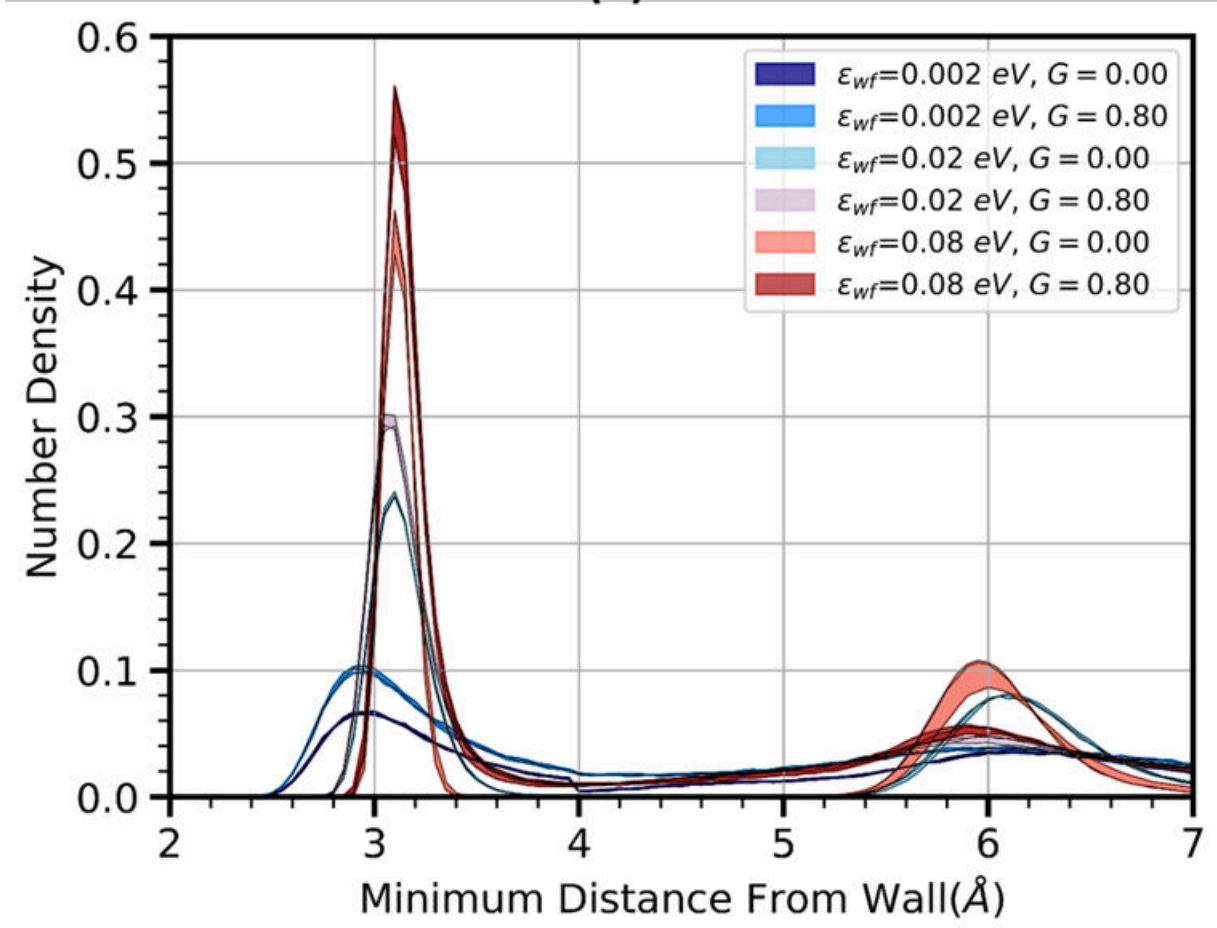

Figure 3 (a) RDF of the liquid atoms with respect to the solid atoms and (b) the number density, using the scheme proposed by Willard and Chandler [26], for different values of the strength of the solid-liquid interactions and depth of surface roughness. For $G=0.8$ the width of each band indicates the variations in the results obtained by different geometries which share the same fractal dimension (the individual cases are shown as black lines within each band). For $G=0$, where there is only one possible geometry, the width corresponds to differences between cases that start at a different microstate. 
Increasing $\varepsilon_{w f}$ also increases the RDF and density of the first peak, as more liquid atoms are attracted to the solid surface. Previous MD simulations have shown that the thermal resistance is inversely proportional to the maximum density of the liquid layer adjacent to a smooth surface [10]. Yet for strong solid-liquid interactions, our results show that the introduction of surface roughness can potentially result in a similar thermal resistance, despite the larger value of the RDF.

In search of alternative physical mechanisms that give rise to these discrepancies, the intrinsic thermal resistance is calculated. Previous investigations normalised the total thermal conductance by the interfacial area [17]. Due to the complexity of the solid surface of the present model, the interfacial area is not well defined, thus we consider the product of the thermal resistance with the number of liquid atoms at the interface for each.

We consider liquid atoms to be part of the interface if they are within a cut off distance of 4 from a solid atom ${ }^{1}$ (Figure $4 \mathrm{a}$ and Figure $4 \mathrm{~b}$ ). Even at low values of $\varepsilon_{w f}$, the number of liquid atoms at the interface is significantly greater for rough surfaces, suggesting a greater effective area (Figure 4b). As the strength of the solid-liquid interaction increases, the number of liquid particles increases continuously. This behaviour is quite different from previous studies that observed a sudden jump in the effective area [18]. This jump corresponds to the transition between the Cassie and Wenzel regimes, i.e. when the solid-liquid interaction overcomes the surface tension of the liquid resulting in the wetting of the grooves. Our geometries, however, are a superposition of a large spectrum of wavenumbers. The smallest wavenumbers correspond to smooth peaks and troughs that are wetted even at low values of $\varepsilon_{w f}$. Yet the roughness corresponding to higher wavenumber roughness remains unwetted. As the solid-liquid interaction strengthens, the liquid atoms start to wet these high wavenumber protrusions. This results in a greater rate of increase of the number of interfacial liquid atoms for the rough surfaces (as observed by the slope of the curves in Figure 4b).

\footnotetext{
${ }^{1}$ The number of atoms at the interface can be obtained from the Figure Error! Main Document Only.b, by multiplying the values of the graph by the normalizing factor (see methodology) and then summing the resulting values, corresponding to the number of particles at a distance from the wall, from 0 to 4
} 
(a)

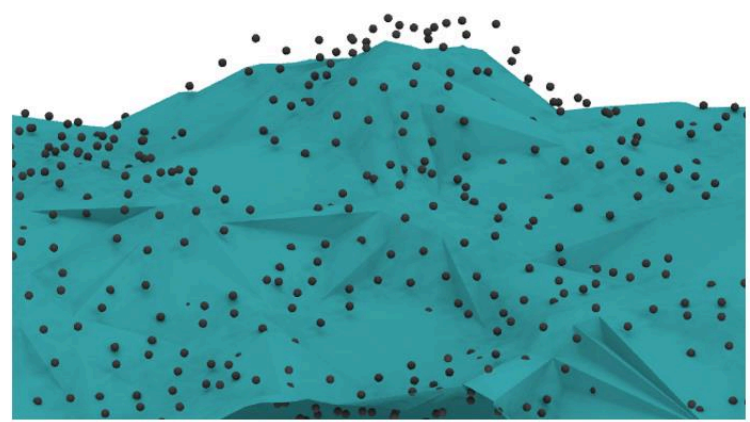

(c)

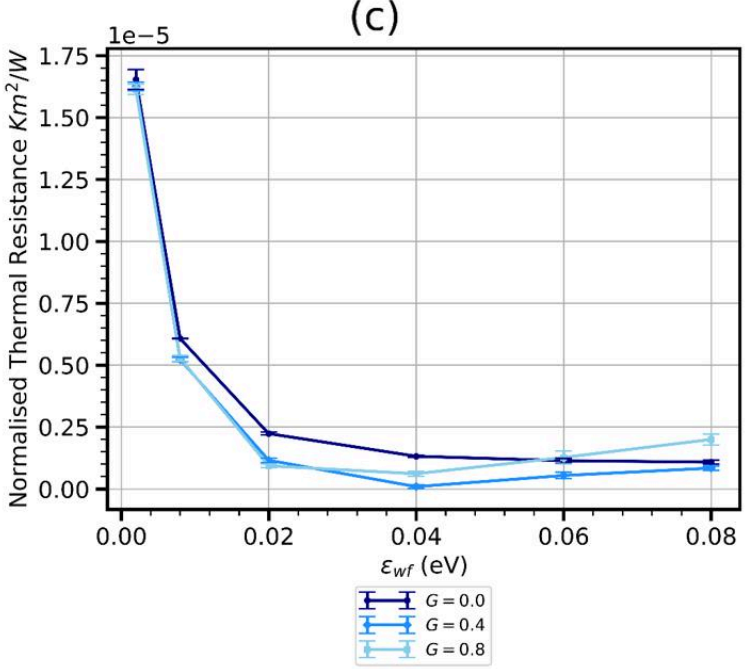

(b)

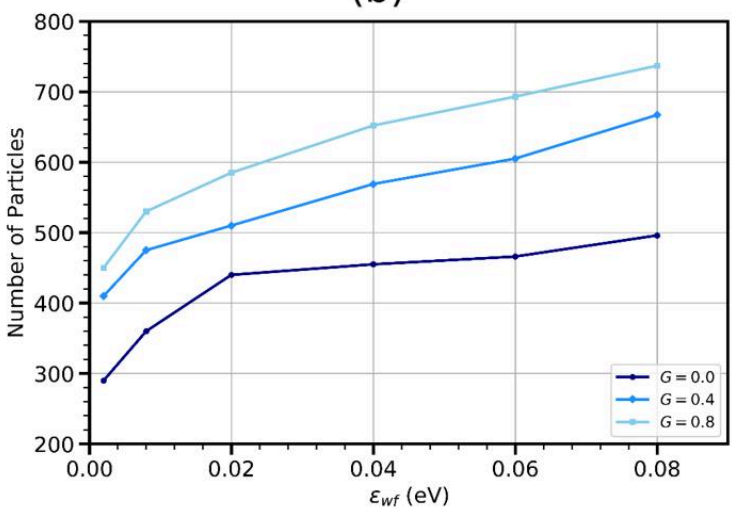

(d)

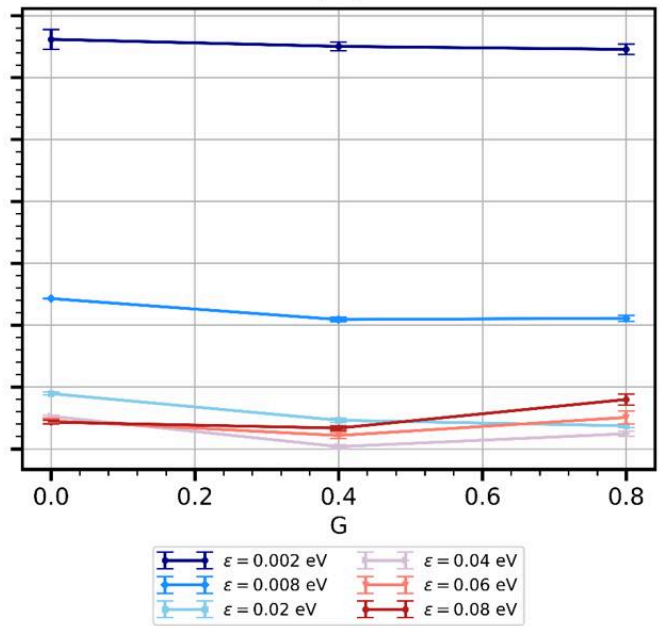

Figure 4 The top row shows (a) a schematic of the liquid particles in the vicinity of the solid interface, and (b) the number of particles at the solid liquid interface, as a function of the strength of the solid-liquid interaction, for different depths of surface roughness. The bottom row shows the thermal resistance multiplied by the number of liquid atoms at the solid-liquid interface for each case, as a function of (c) the strength of the solid-liquid interactions and (d) the average depth of surface roughness.

The normalised thermal resistance (Figure 4c Figure 4d) shows trends similar to those observed in Figure 2: the ITR on smooth surfaces decreases monotonically with increasing $\varepsilon_{w f}$, while for rough surfaces we observe a sharp decrease up to $\varepsilon_{w f}=0.04 \mathrm{eV}$ followed by a minor increase. Unlike the original data, however, the ITR between the different surfaces coincides for weak solid-liquid interactions, i.e. $\varepsilon_{w f}<0.01 \mathrm{eV}$. This suggests that the initially observed differences in thermal resistance were due to the increased effective area exposed by the rough surfaces. For $0.01<\varepsilon_{w f}<0.04 \mathrm{eV}$, the ITR of the rough surfaces decreases below that of the smooth surface. Nevertheless, the thermal resistance for the geometries with $\mathrm{G}=0.4$ and $\mathrm{G}=0.8$ coincides. Past $\varepsilon_{w f}>0.04 \mathrm{eV}$, the ITR for the smooth surface starts to converge, while for $\mathrm{G}=0.4$ and $\mathrm{G}=0.8$ it starts to increase. Furthermore, the rate of increase is higher for $\mathrm{G}=0.8$ than for the shallower roughness. Finally, at $\varepsilon_{w f}=0.08 \mathrm{eV}$, the geometry with the deepest roughness has a thermal resistance that is almost twice that observed on the flat and less rough geometries, the latter two of which have the same value.

Studying the vibrational properties of the solid should also shed light on how the heterogeneities at the solid-liquid interface affect the thermal resistance. We first consider 
the vibrational properties of a wall with a free surface, i.e. in the absence of a liquid. Due to the lack of a second medium, there is no heat transfer across the free surface. We thus use these free-surface cases as a baseline against which the remaining cases can be compared.

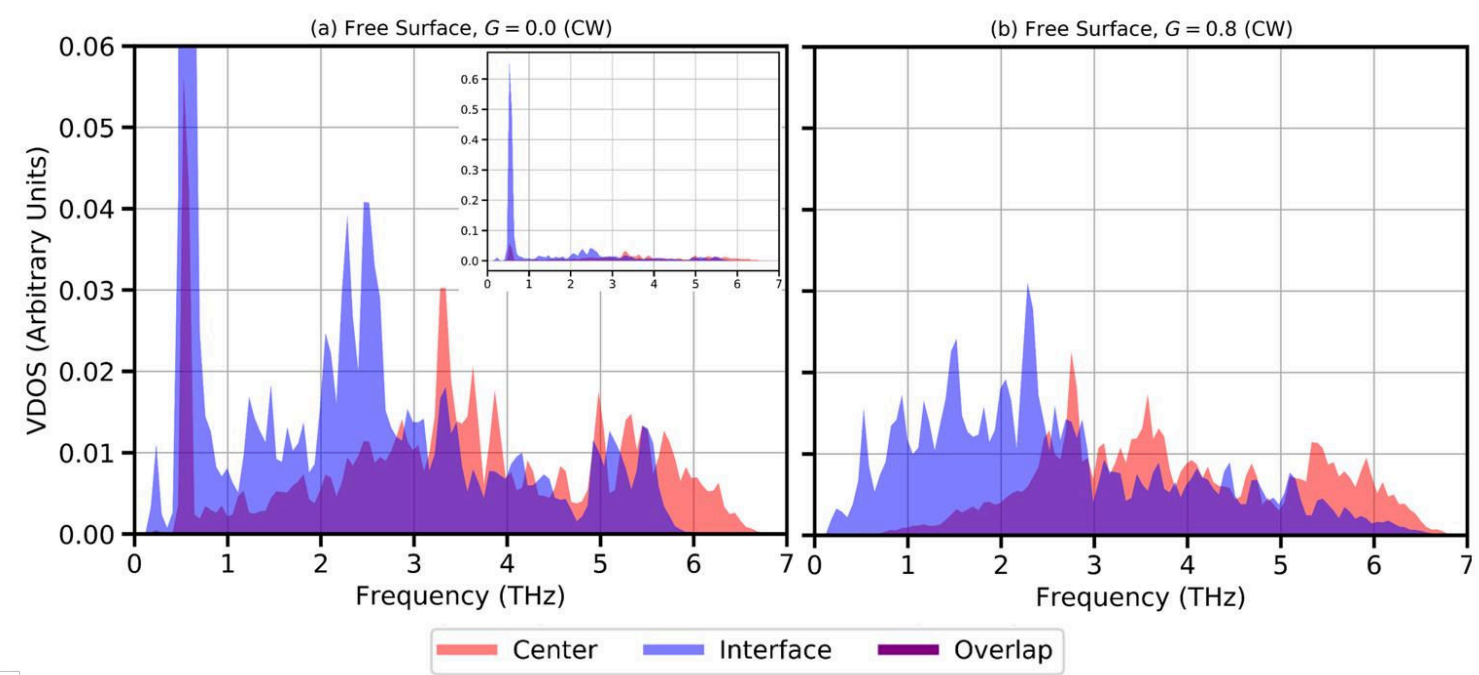

Figure 5 Vibrational Density of States of the silver wall with a free surface, i.e. no liquid next to it, for (a) a smooth and (b) a rough surface. We consider a single plane of atoms at the center (red) of the wall, and a group of atoms at the interface (blue), for various cases. The overlapping region is shaded in purple. The inset in (a) shows the entire range of VDOS for the specific case, while the main figure zooms in on the higher-frequencies, which are common to the remaining cases.

Figure 5 shows a single solid layer at the centre of the wall (red colour), which is considered to be similar to the bulk behaviour of the material, and the solid atoms at a free surface (blue colour). In the absence of surface roughness (Figure $5 \mathrm{a}$ ), we observe a sharp peak at very low frequencies, i.e. approximately $0.5 \mathrm{THz}$. This low frequency peak corresponds to the elastic scattering of phonons against the boundaries of the wall. This is shown by the $y$-component of the HFACF (blue curve in Figure 6a) that oscillates about zero with very little damping. Negative values of the HFACF indicate an inverse correlation, corresponding to phonons propagating in the opposite direction. To verify this, note that the period of the HFACF oscillations (approximately $400 \Delta t=2 p s$ ) matches the time required for a phonon to propagate a across the distance of $50 \AA$, i.e. twice of the wall thickness, when moving at the speed of sound in silver (approximately $2600 \mathrm{~m} / \mathrm{s}$ ). Furthermore, a period of $2 p s$ corresponds to a frequency of $0.5 \mathrm{THz}$, the location of the largest peaks in Figure $5 \mathrm{a}$. Thus in the absence of surface roughness, phonons travel ballistically and bounce of the solid boundaries with little loss of energy.

In the interfacial layer we observe frequency bands that are slightly red-shifted and have a larger amplitude than the central plane, e.g., at $2.5 \mathrm{THz}$ in Figure 5a. This suggests localisation of phonons, i.e. phonons that are spatially localised in the vicinity of heterogeneities, which impede the flow of heat [31, 32].

The morphology of a solid can alter its vibrational properties [33]. As we introduce surface roughness (Figure $5 b$ ), the low frequency band observed in the smooth surface practically vanishes. On the rough surface, we also observe a wider band between $1 \mathrm{THz}$ and $2.5 \mathrm{THz}$, indicative of anharmonic scattering [34, 35]. This behaviour can also be seen by the HFACF (blue curve in Figure 6b), where the oscillatory pattern is short-lived and very irregular, suggesting a destructive superposition of different frequencies. Finally, the introduction of 
surface roughness seems to significantly reduce the VDOS of the longitudinal branch the interface.
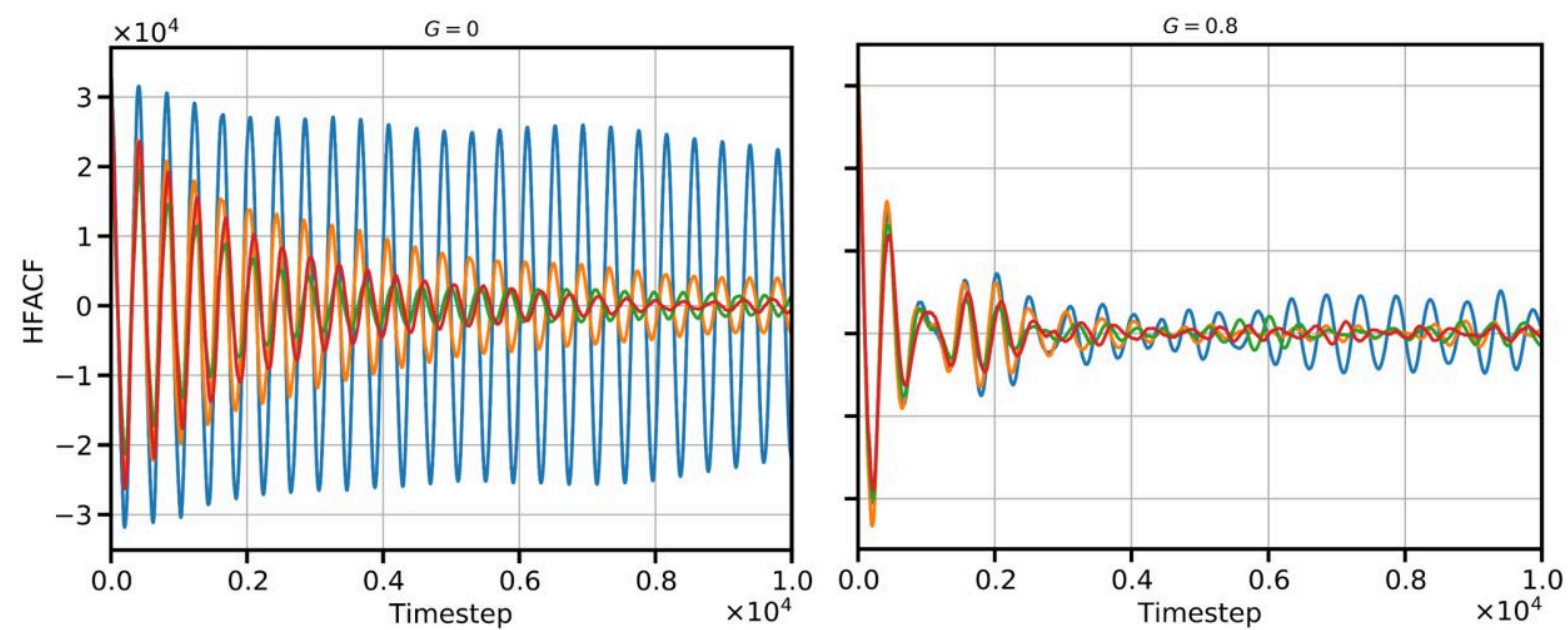

- Free Surface $-\varepsilon_{w f}=0.002 \mathrm{eV}-\varepsilon_{w f}=0.04 \mathrm{eV}-\varepsilon_{w f}=0.08 \mathrm{eV}$

Figure 6 The $y$-component of the Heat Flux Autocorrelation Function (HFACF) plotted against time for (a) $G=0$ and $(b) \mathrm{G}=$ 0.8

In the presence of liquid and weak solid-liquid interactions (i.e. $\varepsilon_{w f}=0.002 \mathrm{eV}$ ), the VODS look pretty similar to the free-surface cases for both the smooth and rough walls (cf. Figure $7 \mathrm{a}$ and Figure $7 \mathrm{~b}$ to Figure $5 \mathrm{a}$ and Figure $5 \mathrm{~b}$ ). The main difference is that on smooth surfaces with $\varepsilon_{w f}=0.002 \mathrm{eV}$ we also observe localisation of longitudinal phonons (see blue peak at $\approx 5.2 \mathrm{THz}$ in Figure $7 \mathrm{a}$ ). The similar nature with the VDOS on free surfaces suggests that the solid and liquid atoms oscillate independently of each other, and explains the high thermal resistance at $\varepsilon_{w f}=0.002 \mathrm{eV}$.

As the strength of the solid-liquid interaction increases, the vibrational bands at the interface of both the smooth and rough walls, start to match the amplitudes and frequencies of the central layer; cf. Figure 7a, c, and e for smooth surface, and Figure $7 b, d$ and $f$ for rough surfaces. This delocalisation of phonons suggests better vibrational coupling at the interface $[28,27,36]$, and the reason behind the decrease of the thermal resistance when increasing $\varepsilon_{w f}$, even after the interfacial liquid atoms are accounted for (Figure 4). This is also shown by the HFACF, where with increasing $\varepsilon_{w f}$, the oscillatory patterns dissipate faster (Figure 6a and b), as phonons percolate into the liquid.

Finally, at $\varepsilon_{w f}=0.04 \mathrm{eV}$, the smooth wall shows localised phonon modes at approximately $1.8 \mathrm{THz}$, which are absent on VODS of the rough wall. This could explain the marginally lower thermal resistance in the presence of roughness. On the contrary, we believe that the increase of the thermal resistance for cases with rough walls and values of $\varepsilon_{w f}>0.04 \mathrm{eV}$ is due to a minor increase in phonon localisation. Specifically, for $\varepsilon_{w f}=0.08 \mathrm{eV}$ we observe two small peaks, one at approximately $1.9 \mathrm{THz}$ and one at approximately $2.5 \mathrm{THz}$, that are absent at $\varepsilon_{w f}=0.04 \mathrm{eV}$. 


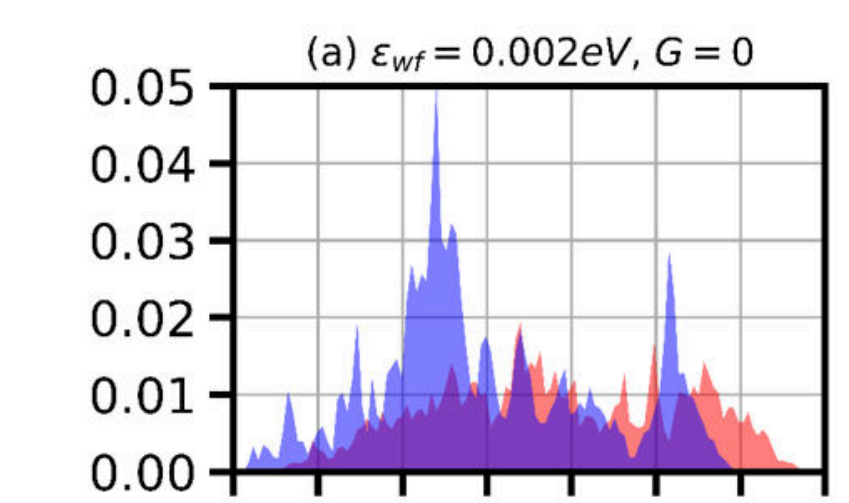

(b) $\varepsilon_{w f}=0.002 \mathrm{eV}, G=0.8$
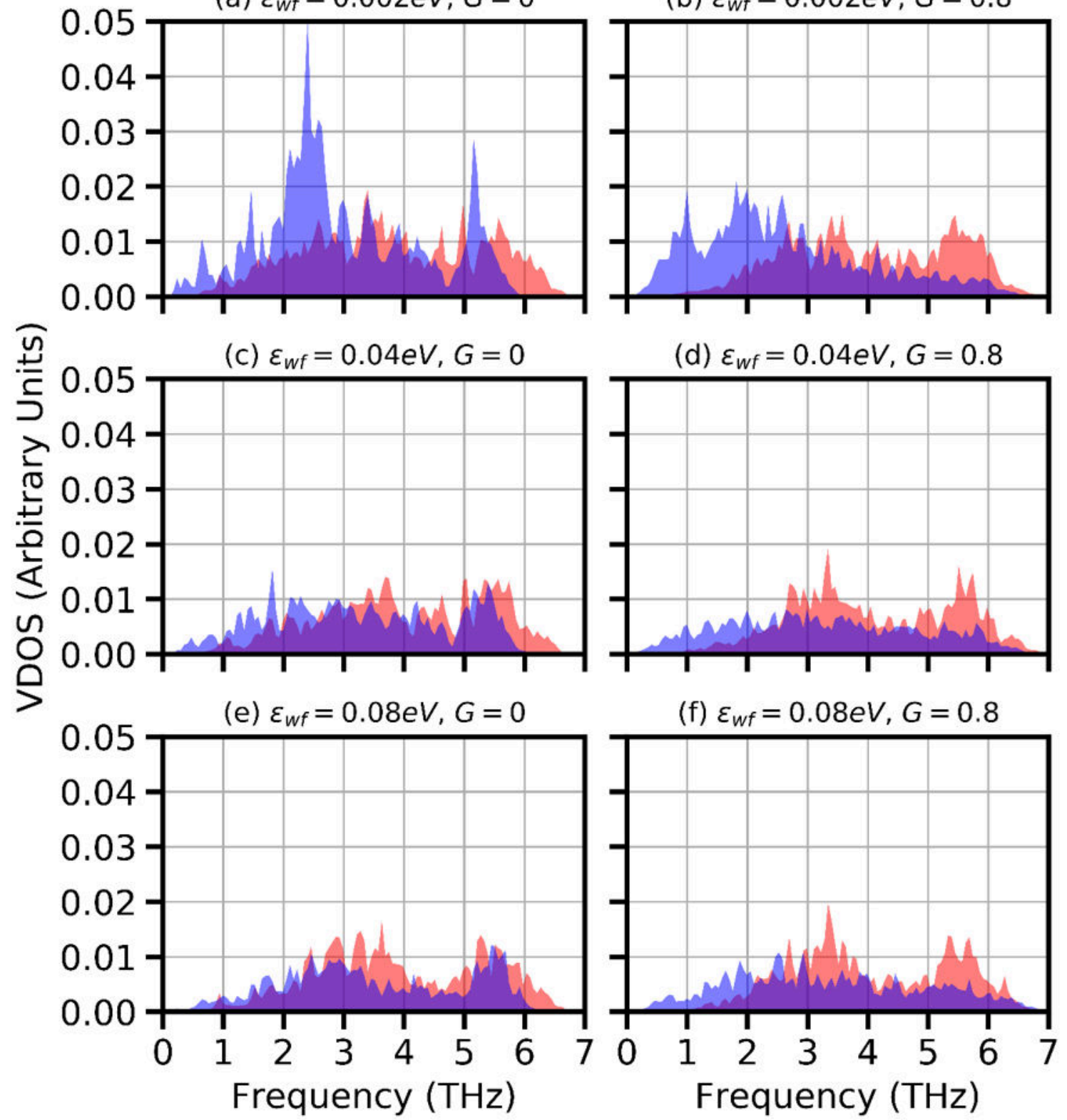

Center

Interface

(d) $\varepsilon_{w f}=0.04 \mathrm{eV}, G=0.8$

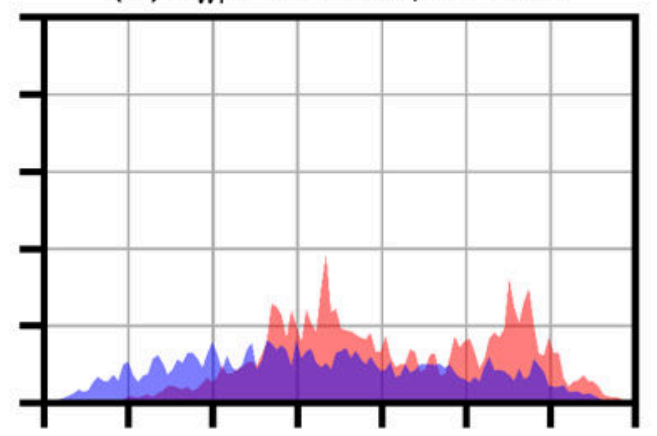

(f) $\varepsilon_{w f}=0.08 \mathrm{eV}, G=0.8$

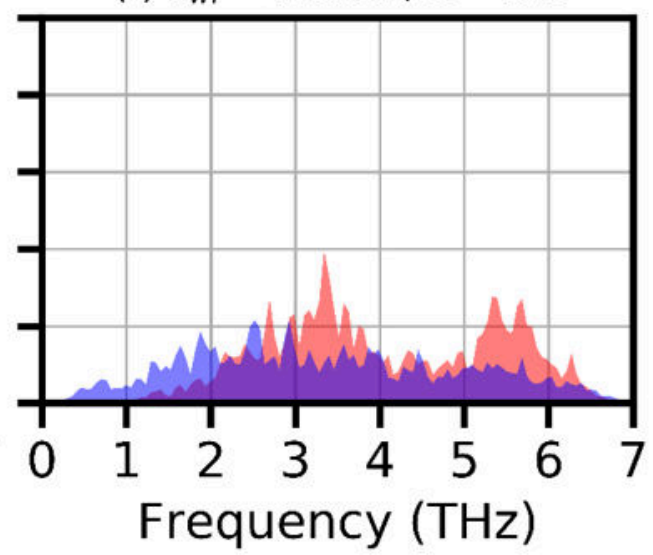

Figure 7 Vibrational Density of States of a single plane of atoms at the center (red) of the wall, and a group of atoms at the interface (blue), for various cases. The overlapping region is shaded in purple. We present the VDOS for different cases: (a) $\varepsilon_{w f}=0.002 \mathrm{eV}$ and $G=0$, (b) $\varepsilon_{w f}=0.002 \mathrm{eV}$ and $G=0.8$, (c) $\varepsilon_{w f}=0.04 \mathrm{eV}$ and $G=0$, (d) $\varepsilon_{w f}=0.04 \mathrm{eV}$ and $G=$ 0.8 , (e) $\varepsilon_{w f}=0.08 \mathrm{eV}$ and $G=0$, and $(f) \varepsilon_{w f}=0.08 \mathrm{eV}$ and $G=0.8$.

A previous investigation has also found an optimal value for $\varepsilon_{w f}$ beyond which the thermal resistance increases [37]. They attributed the phenomenon to the thermal resistance between parallel liquid layers, which become more distinct and further apart. While we did not observe this increase on smooth channels, we also believe that the increase in ITR past $\varepsilon_{w f}=0.04 \mathrm{eV}$ is due to an adaptation of the liquid structure.

The Kapitza resistance has previously been calculated as a function of the maximum value of the first density peak next to the solid walls [10]. Through a regression analysis of our results, 
we found the thermal resistance to behave similar to the square root of $\frac{1}{g_{1}(r)^{2} \times \varepsilon_{w f}}$, where $g_{1}(r)$ is the RDF of the first density peak (see Figure 8). Specifically:

$$
R_{k, \text { mean }}=1.3 \times 10^{-10} \sqrt{\frac{1}{g_{1}(r)^{2} \times \varepsilon_{w f}}+\left(300 \varepsilon_{w f} G\right)},
$$

The first term of the regression tries to capture the rapid decay in the thermal resistance observed at each surface, as the value of $\varepsilon_{w f}$ (and subsequently $g_{1}(r)$ ) increases. The second term attempts to capture the smaller effects of roughness, that seem to depend on the strength of the solid-liquid interactions. The regression seems to capture the large-scale features and trends of the data. Yet it doesn't always capture small variations, particularly at the lower values of the $\mathrm{x}$-axis in Figure 8.

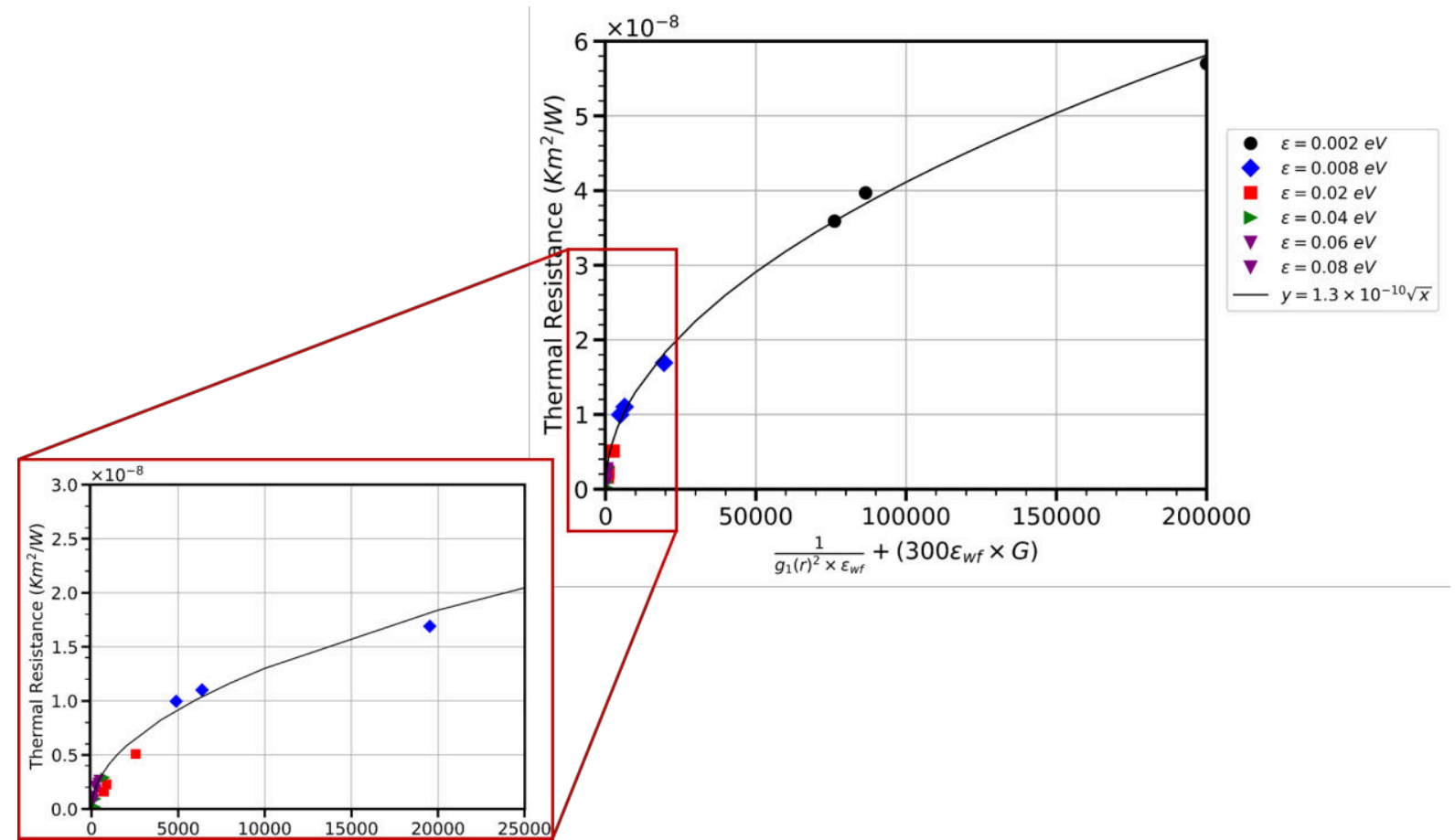

Figure 8 Thermal resistance as a function of the height of the first peak of the RDF, the strength of the solid-liquid interaction, and the depth of surface roughness. The markers correspond to the MD data while the straight line is a regression line.

As a final remark, the thermal resistance between the "hot" and "cold" surfaces generally differs (Figure 9). A maximum of approximately $6 \%$ difference is observed between the hot and cold wall for the case of a smooth wall with $\varepsilon_{w f}=0.002 \mathrm{eV}$. The temperature dependence has been previously observed by others, but with contradicting conclusions. Some studies show a higher thermal resistance on the "cold" wall [1, 10], while others show a higher thermal resistance on the "hot" wall $[2,38,5]$. These discrepancies have been credited to the type of liquid molecules considered, with monatomic molecules encompassing a higher thermal resistance on the cold wall , while polar molecules give a higher thermal resistance on the cold wall [10]. In the present study, both behaviours have been observed depending on the strength of the solid-liquid interaction. For low values of $\varepsilon_{w f}$ the thermal resistance is greater at the cold wall. At the higher values of $\varepsilon_{w f}$ considered here, the thermal resistance seems to be the same between the hot and cold wall and, in some cases, even marginally greater (see $\varepsilon_{w f}=0.002 \mathrm{eV}$ at $G=0$ ). Finally, the difference in 
thermal resistance between the hot and cold walls seems to quickly diminish as we introduce and increase the depth of surface roughness.

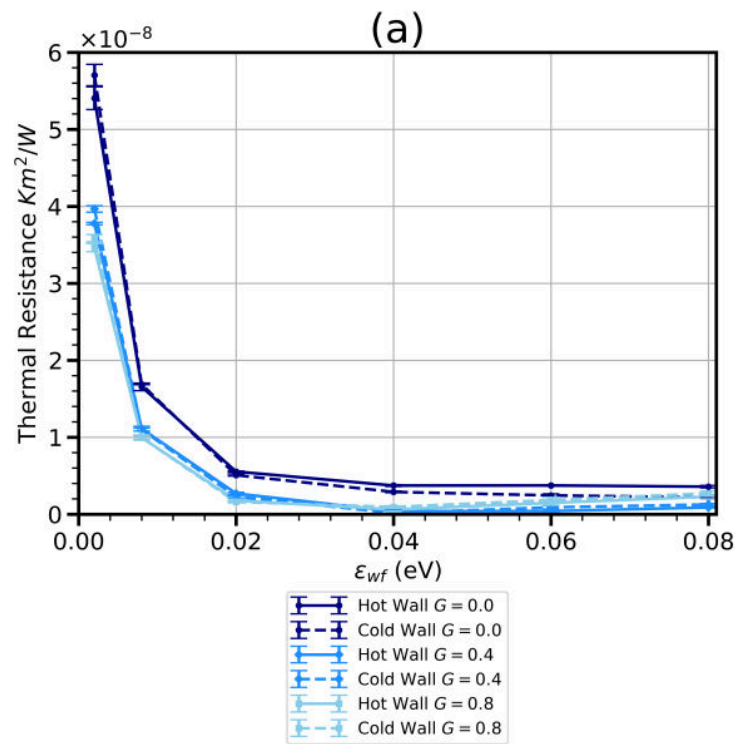

(b)

Figure 9 Comparison of the thermal resistance between the hot (solid line) and cold (dashed line) wall as a function of (a) the strength of the solid-liquid interactions and (b) the depth of surface roughness

\section{Conclusions}

The effects of the depth of surface roughness and strength of the solid-liquid interaction on ITR were investigated using MD. The main findings are summarised below:

1. On smooth surfaces, the ITR decreases as the strength of the solid-liquid interaction increases. Similar trends have been observed in the literature for different liquids and solids.

2. On rough surfaces, the ITR initially decreases in a fashion similar to the decrease observed in the smooth cases. As the strength of the solid-liquid interaction increases further the thermal resistance starts to increase slightly.

3. The effect of surface roughness on ITR depends on the strength of the solid-liquid interaction. For weak interactions, increasing surface roughness results in a significant decrease in ITR. For strong interactions, increasing the surface roughness doesn't affect the thermal resistance as much, and in some cases results in a minor increase.

4. The inconsistent effect of surface roughness is due to two separate phenomena. Firstly, the increasing depth of surface roughness implies an increase in the area of the solid surface that is in contact with the liquid, thus reducing ITR. Secondly, surface roughness and the strength of the solid-liquid interaction alters vibrational properties of materials, introducing anharmonic effects and phonon localization, thus affecting ITR.

5. Using regression analysis, an empirical formula for the ITR as a function of the average depth of surface roughness and the strength of the solid-liquid interaction, has been proposed.

6. The ITR has minor differences between the hot and cold wall. While most previous studies consistently observe either larger or lower thermal resistance on the cold wall, we observe both behaviours, depending on the strength of the solid-liquid interaction. 
For weak interactions, the cold wall has a larger ITR while for strong interactions, the cold wall has a marginally lower ITR.

7. As we introduce and increase the depth of surface roughness, the differences between the hot and cold wall diminish.

8. Completely different geometries sharing the same fractal dimension and depth of surface roughness result in practically the same ITR and RDF. This suggests that these parameters are sufficient to describe correlations between ITR and surface geometry, regardless of the precise molecular configuration.

\section{Conflicts of interest}

There are no conflicts of interest to declare

\section{Acknowledgments}

Results were obtained using the ARCHIE-WeSt High Performance Computer (www.archiewest.ac.uk) based at the University of Strathclyde.

\section{References}

[1] G. L. Pollack, “Kapitza resistance," Reviews of Modern Physics, vol. 4, no. 1, p. 48, 1969.

[2] E. T. Swartz and R. O. Pohl, "Thermal boundary resistance," Reviews of modern physics, vol. 61, no. 3, p. 605, 1989.

[3] M. Frank and D. Drikakis, "Solid-like heat transfer in confined liquids," Microfluidics and Nanofluidics, vol. 21, no. 9, p. 148, 2017.

[4] M. Frank and D. Drikakis, "Thermodynamics at Solid--Liquid Interfaces," Entropy, vol. 20, no. 5, p. 2018, 2018.

[5] B. H. Kim, A. Beskok and T. Cagin, "Molecular dynamics simulations of thermal resistance at the liquid-solid interface," The Journal of chemical physics, vol. 129, no. 17, p. 174701, 2008.

[6] L. Xue, P. Keblinski, S. Phillpot, S.-S. Choi and J. Eastman, "Two regimes of thermal resistance at a liquid--solid interface," The Journal of Chemical Physics, vol. 118, pp. 337$-339,2003$.

[7] J.-L. a. C. F. Barrat, "Kapitza resistance at the liquid-solid interface," Molecular Physics, vol. 101, pp. 1605--1610, 2003.

[8] B. Kim, "Thermal resistance at a liquid--solid interface dependent on the ratio of thermal oscillation frequencies," Chemical Physics Letters, vol. 554, pp. 77--81, 2012. 
[9] M. Frank, M. Kio, D. Drikakis, L. Könözsy and N. Asproulis, "Mass and Stiffness Effects on Thermal Resistance at the Solid-Liquid Interface of Nanofluidic Channels," Journal of Computational and Theoretical Nanoscience, vol. 15, pp. 141-146, 2018.

[10] D. Alexeev, J. Chen, J. H. Walther, K. P. Giapis, P. Angelikopoulos and P. Koumoutsakos, "Kapitza resistance between few-layer graphene and water: Liquid layering effects," Nano letters, vol. 15, no. 9, pp. 5744--5749, 2015.

[11] R. N. Wenzel, "Surface roughness and contact angle," The Journal of Physical Chemistry, vol. 53, no. 9, pp. 1466--1467, 1949.

[12] R. N. Wenzel, "Resistance of solid surfaces to wetting by watter," Industrial \& Engineering Chemistry, vol. 28, no. 8, pp. 988-994, 1936.

[13] J. Mittal and G. Hummer, "Interfacial thermodynamics of confined water near molecularly rough surfaces," Faraday discussions, vol. 146, pp. 341--352, 2010.

[14] F. Leroy and F. Muller-Plathe, "Solid-liquid surface free energy of Lennard-Jones liquid on smooth and rough surfaces computed by molecular dynamics using the phantomwall method," The Journal of chemical physics, vol. 133, no. 4, p. 044110, 2010.

[15] H. Hu and Y. Sun, "Effect of nanopatterns on Kapitza resistance at a water-gold interface during boiling: A molecular dynamics study," Journal of Applied Physics, vol. 112, p. 053508, 2012.

[16] Y. Chen and C. Zhang, "Role of surface roughness on thermal conductance at liquid-solid interfaces," International Journal of Heat and Mass Transfer, vol. 78, no. 1, pp. 624$-629,2014$.

[17] H. Acharya, N. J. Mozdzierz, P. Keblinski and S. Garde, "How chemistry, nanoscale roughness, and the direction of heat flow affect thermal conductance of solid--water interfaces," Industrial \& Engineering Chemistry Research, vol. 51, no. 4, pp. 1767--1773, 2011.

[18] D. Surblys, Y. Kawagoe, M. Shibahara and T. Ohara, "Molecular dynamics investigation of surface roughness scale effect on interfacial thermal conductance at solid-liquid interfaces," The Journal of Chemical Physics, vol. 150, no. 11, p. 114705, 2019.

[19] A. Cassie and S. Baxter, "Wettability of porous surfaces," Transactions of the Faraday society, vol. 40, pp. 546--551, 1944.

[20] M. Papanikolaou, M. Frank and D. Drikakis, "Effects of surface roughness on shear viscosity," Physical Review E, vol. 95, no. 3, p. 033108, 2017.

[21] M. Papanikolaou, M. Frank and D. Drikakis, "Nanoflow over a fractal surface," Physics of Fluids, vol. 28, no. 8, p. 082001, 2016. 
[22] M. Ausloos and D. Berman, "A multivariate Weierstrass-Mandelbrot function," Proceedings of the Royal Society of London A: Mathematical, Physical and Engineering Sciences, vol. 400, no. 1819, pp. 331--350, 1985.

[23] S. Plimpton, "Fast parallel algorithms for short-range molecular dynamics," Journal of computational physics, vol. 117, no. 1, pp. 1--19, 1995.

[24] C. A. Becker, F. Tavazza, Z. T. Trautt and R. A. B. de Macedo, "Considerations for choosing and using force fields and interatomic potentials in materials science and engineering," Current Opinion in Solid State and Materials Science, vol. 17, no. 6, pp. 277--283, 2013.

[25] B. H. Kim, A. Beskok and T. Cagin, "Thermal interactions in nanoscale fluid flow: molecular dynamics simulations with solid--liquid interfaces," Microfluidics and Nanofluidics, vol. 5, no. 4, pp. 551--559, 2008.

[26] A. P. Willard and D. Chandler, "Instantaneous liquid interfaces," The Journal of Physical Chemistry B, vol. 114, no. 5, pp. 1954--1958, 2010.

[27] J. C. Duda, T. S. English, E. S. Piekos, W. A. Soffa, L. V. Zhigilei and P. E. Hopkins, "Implications of cross-species interactions on the temperature dependence of Kapitza conductance," Physical Review B, vol. 19, no. 84, p. 193301, 2011.

[28] M. E. Caplan, A. Giri and P. E. Hopkins, "Analytical model for the effects of wetting on thermal boundary conductance across solid/classical liquid interfaces," The Journal of Chemical Physics, vol. 140, no. 15, 2014.

[29] M. Papanikolaou and K. Salonitis, "Fractal roughness effects on nanoscale grinding," Applied Surface Science, vol. 467, pp. 309--319, 2010.

[30] M. V. Athawale, S. N. Jamadagni and S. Garde, "How hydrophobic hydration responds to solute size and attractions: Theory and simulations," The Journal of chemical physics, vol. 131, no. 11, p. 09B615, 2009.

[31] S. Estreicher, T. Gibbons, B. Kang and M. Bebek, "Phonons and defects in semiconductors and nanostructures: Phonon trapping, phonon scattering, and heat flow at heterojunctions," Journal of Applied Physics, vol. 115, no. 1, p. 012012, 2014.

[32] G. P. Srivastava, The physics of phonons, CRC press, 1990.

[33] G. A. Narvaez, J. Kim and J. W. Wilkins, "Effects of morphology on phonons in nanoscopic silver grains," Physical Review B, vol. 72, no. 15, p. 155411, 2005.

[34] H. Euchner, S. Pailhes, L. Nguyen, W. Assmus, F. Ritter, A. Haghighirad, Y. Grin, S. Paschen and $M$. de Boissieu, "Phononic filter effect of rattling phonons in the thermoelectric clathrate Ba 8 Ge 40+ x Ni 6- x," Physical review B, vol. 86, no. 22, p. 224303, 2012. 
[35] S. Pailhes, H. Euchner, V. Giordano, R. Debord, A. Assy, S. Gomes, A. Bosak, D. Machon, S. Paschen and M. De Boissieu, "Localization of propagative phonons in a perfectly crystalline solid," Physical review letters, vol. 113, no. 2, p. 025506, 2014.

[36] B. Ramos-Alvarado, S. Kumar and G. Peterson, "Solid--Liquid Thermal Transport and Its Relationship with Wettability and the Interfacial Liquid Structure," The journal of physical chemistry letters, vol. 7, pp. 3497--3501, 2016.

[37] C. Wang, J. Chen, J. Shiomi and S. Maruyama, "A study on the thermal resistance over solid--liquid--vapor interfaces in a finite-space by a molecular dynamics method," International Journal of Thermal Sciences, vol. 46, no. 12, pp. 1203--1210, 2007.

[38] M. Barisik and A. Beskok, "Boundary treatment effects on molecular dynamics simulations of interface thermal resistance," Journal of Computational Physics, vol. 231, no. 23, pp. 7881--7892, 2012. 

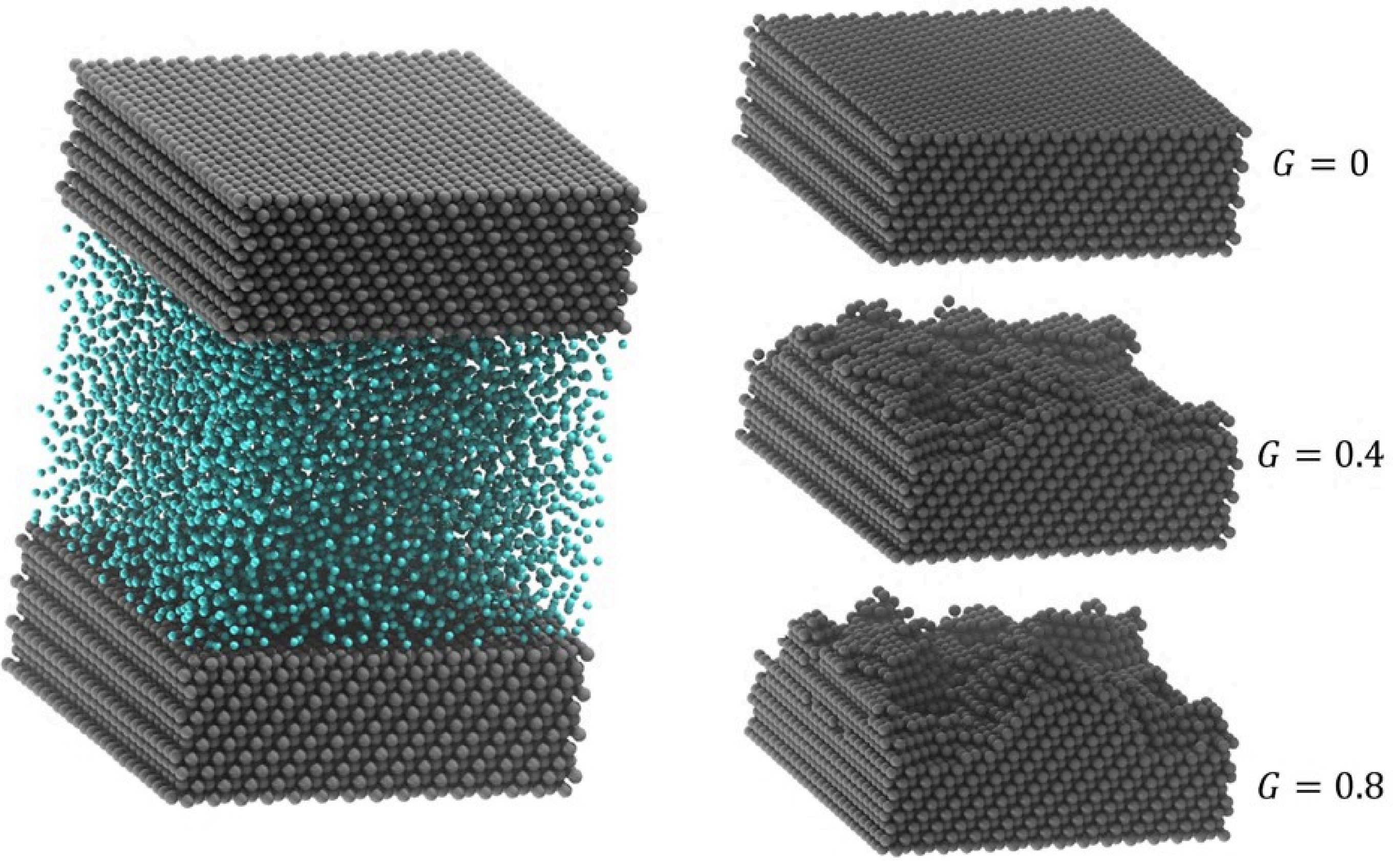


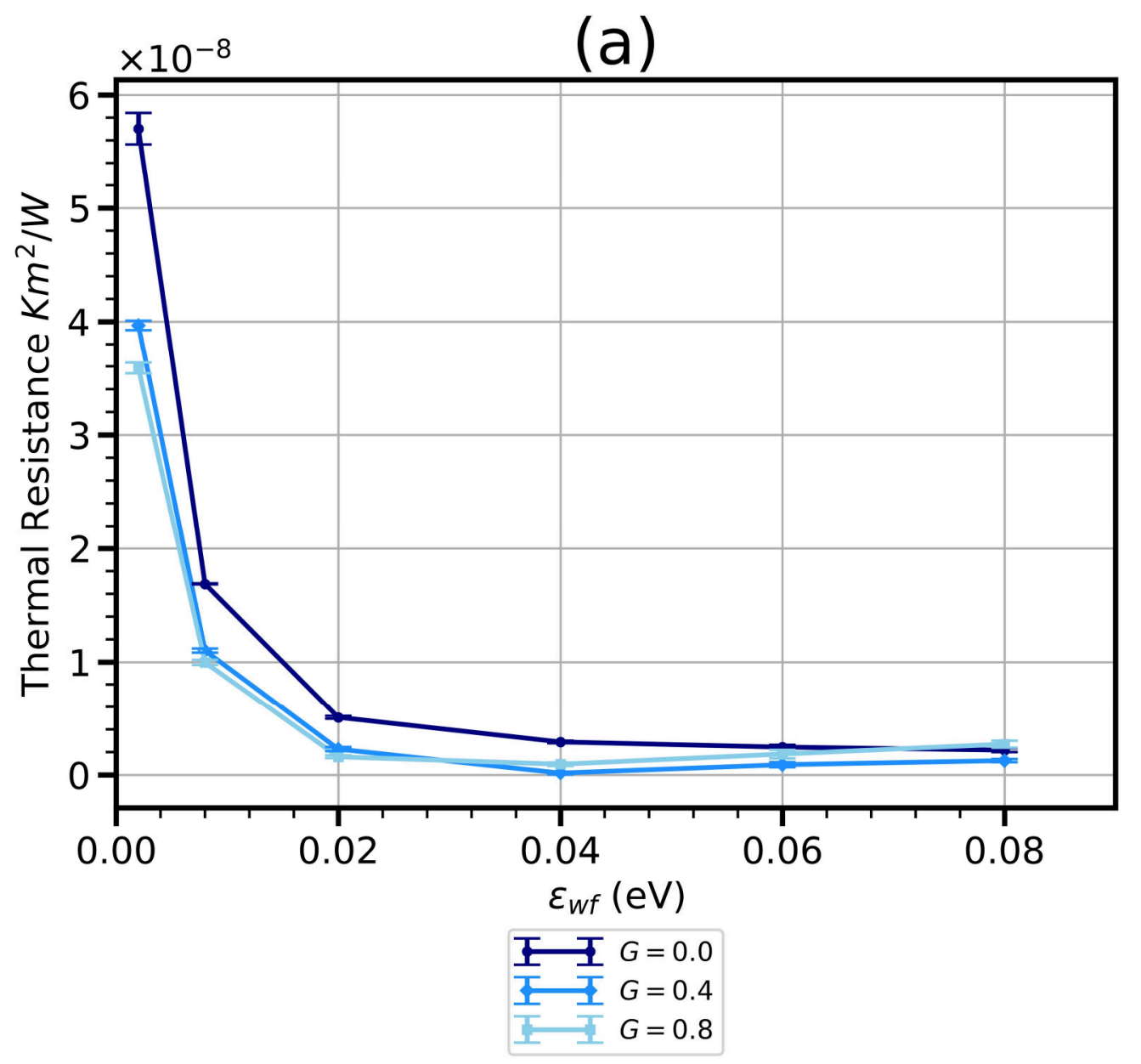

(b)

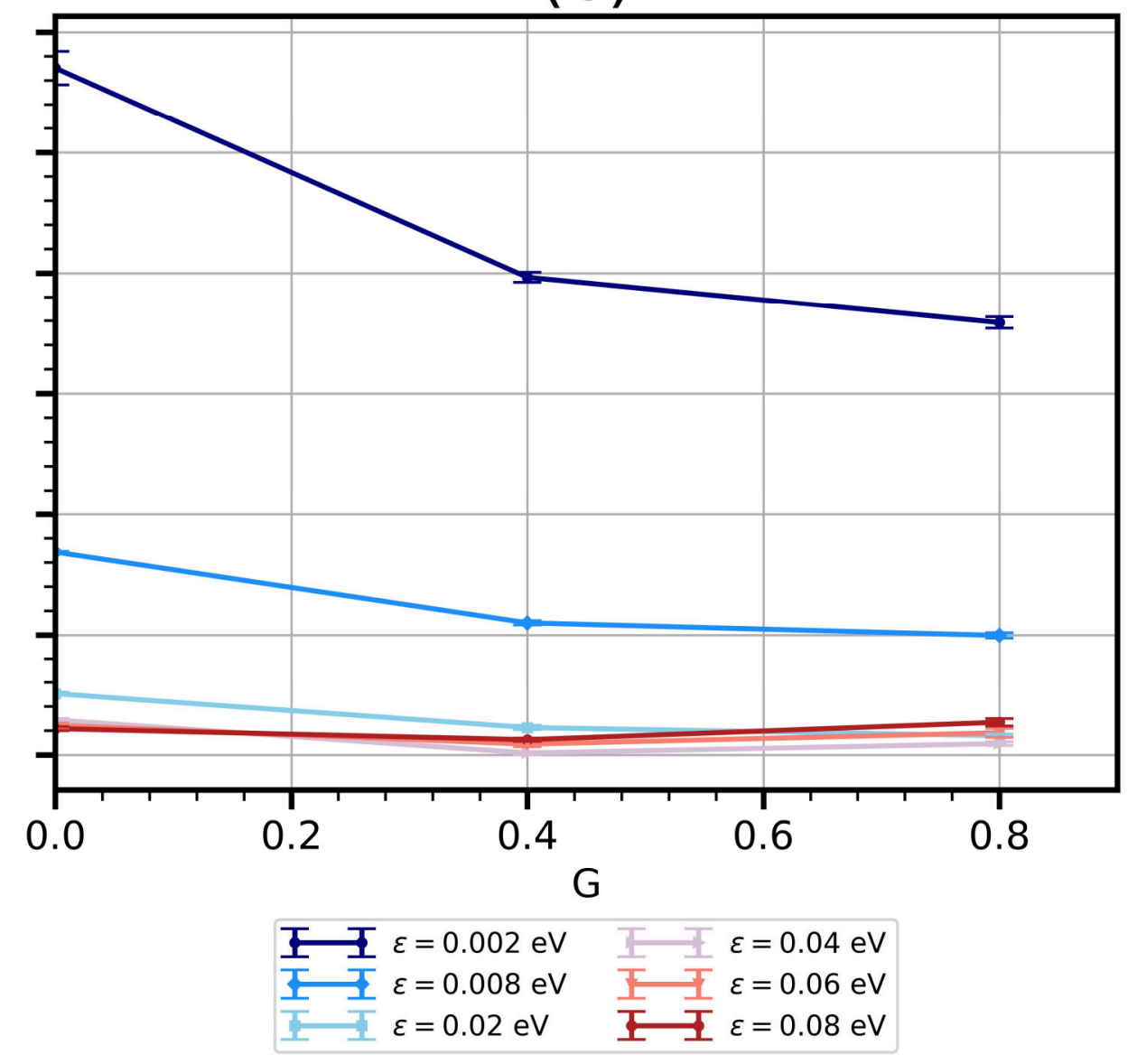


(a)
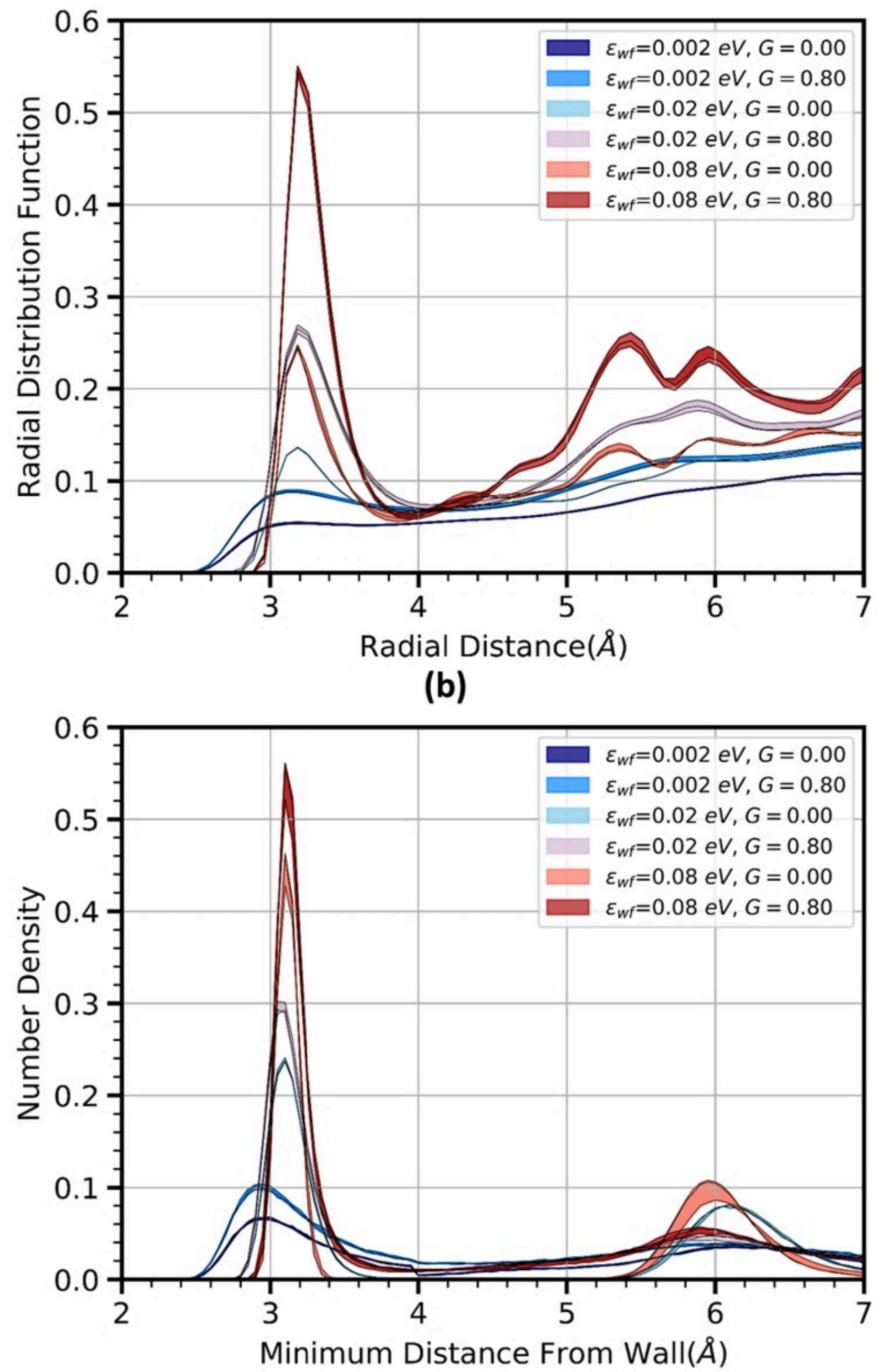
(a)

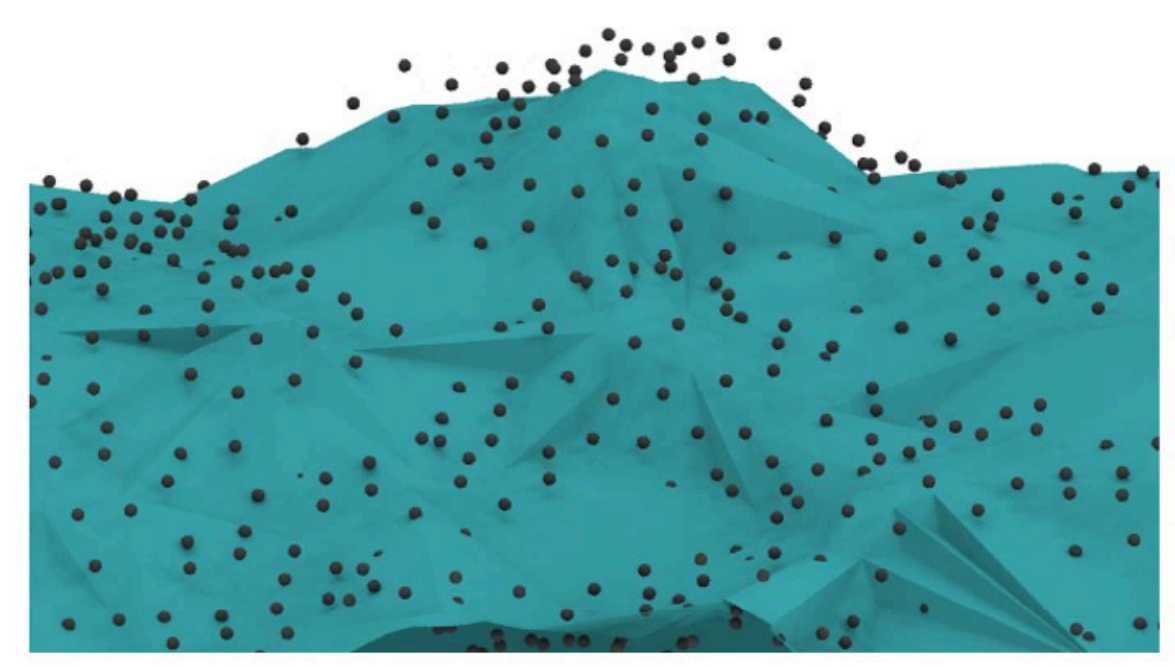

(c)

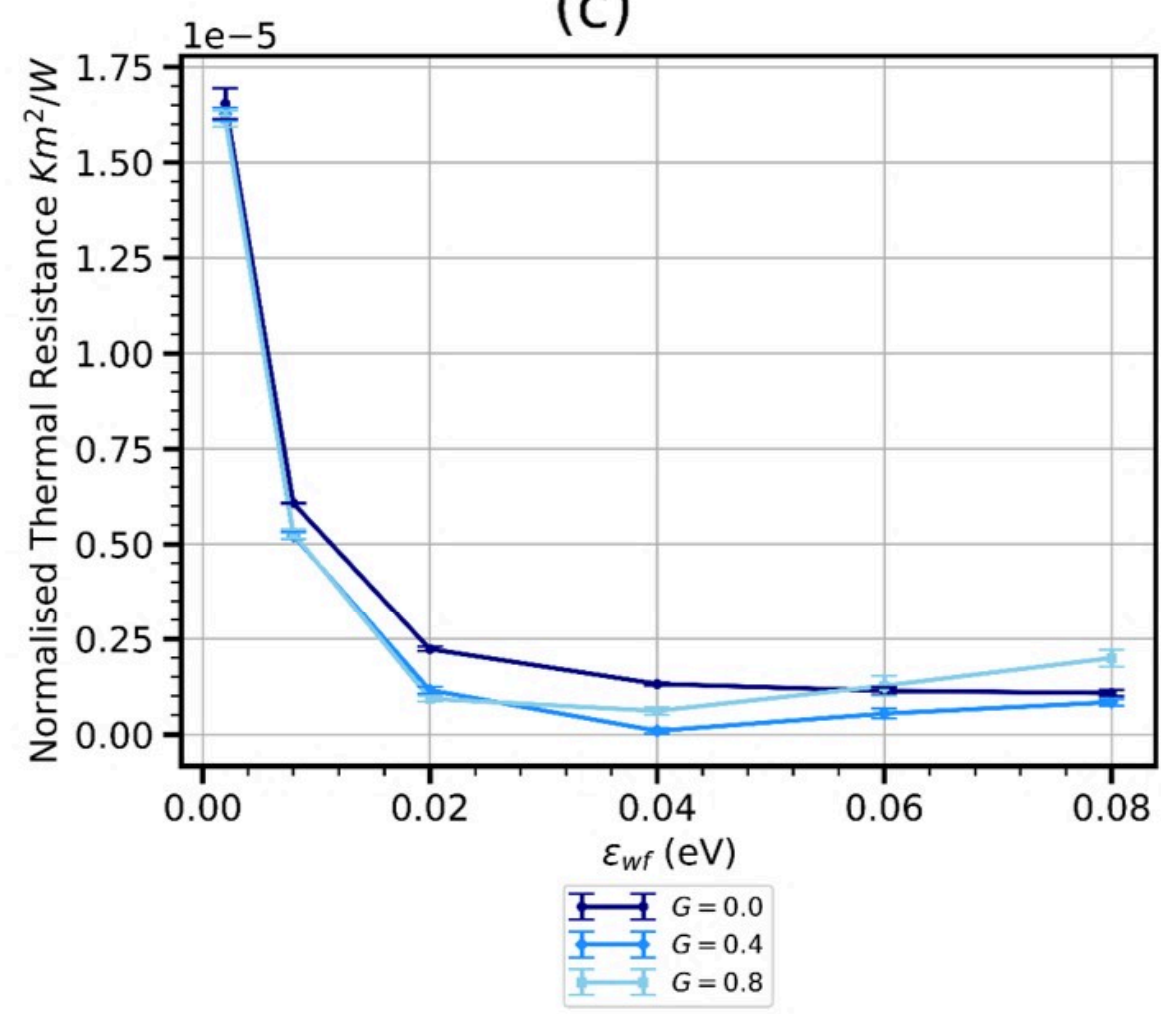

(b)

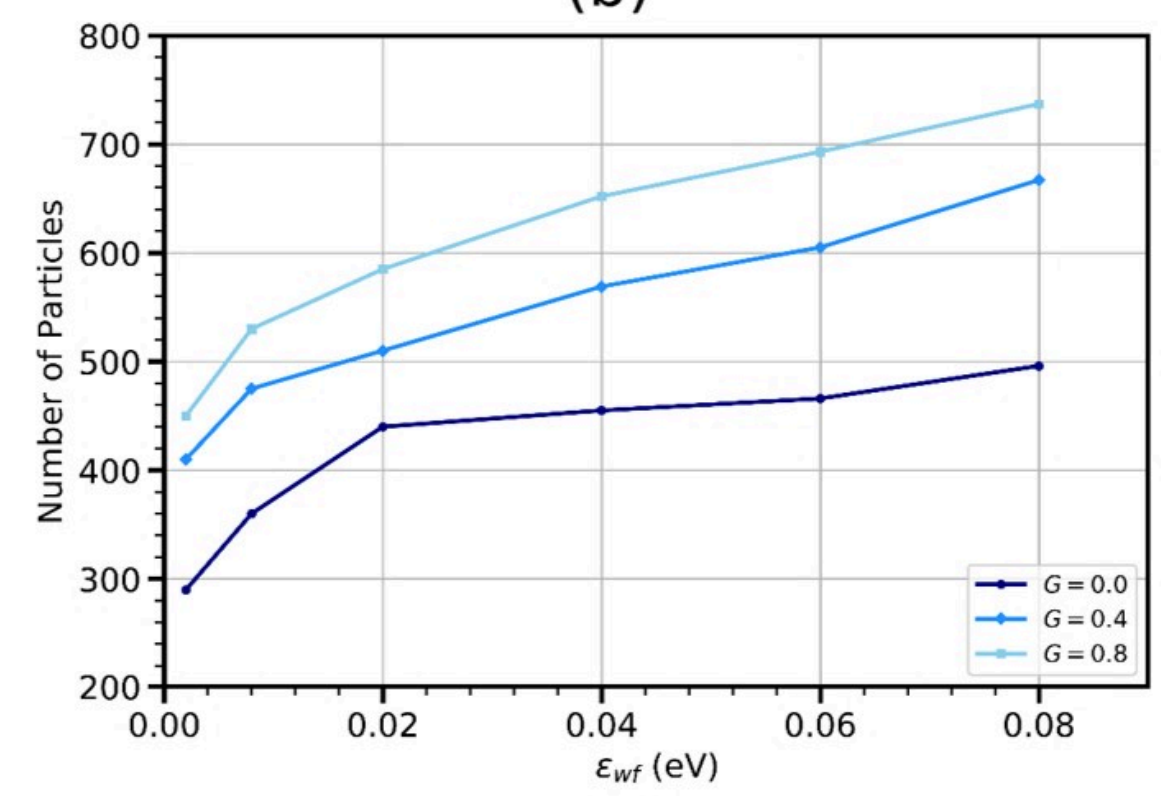

(d)

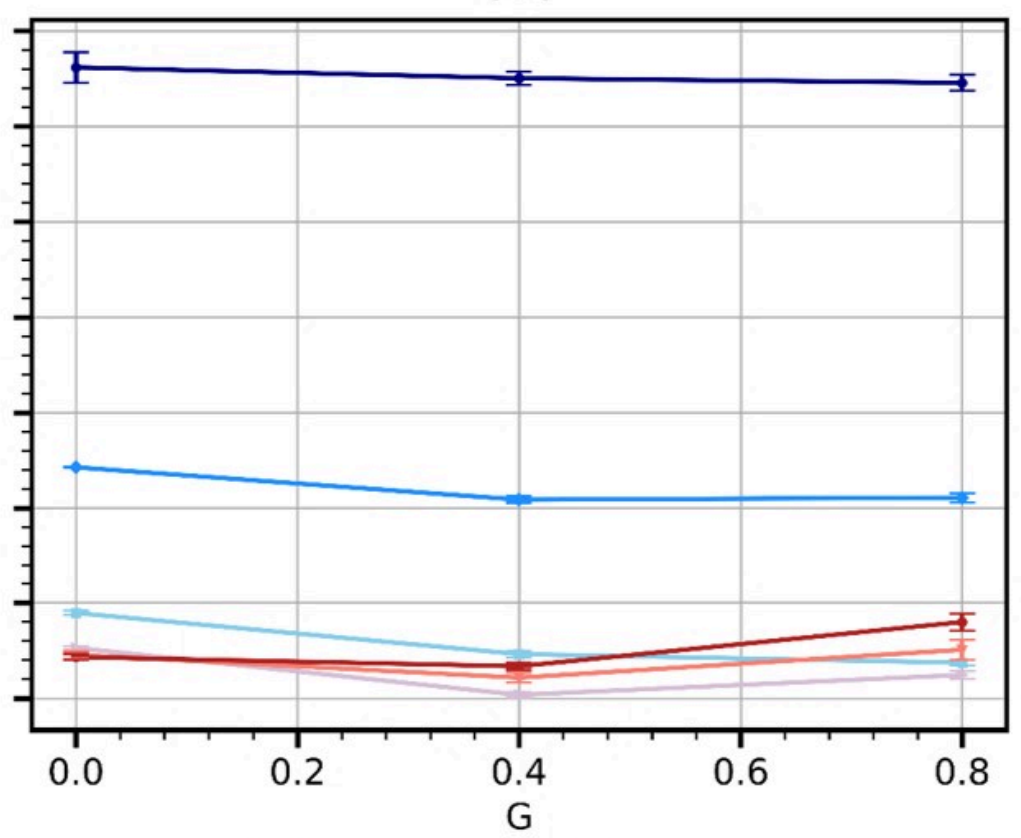

ㅍ $\varepsilon=0.002 \mathrm{eV}$ I $\varepsilon=0.04 \mathrm{eV}$ ㅍ $\varepsilon=0.008 \mathrm{eV}$ I $\varepsilon=0.06 \mathrm{eV}$ ㅍ $\varepsilon=0.02 \mathrm{eV}$ 工王 $\varepsilon=0.08 \mathrm{eV}$ 

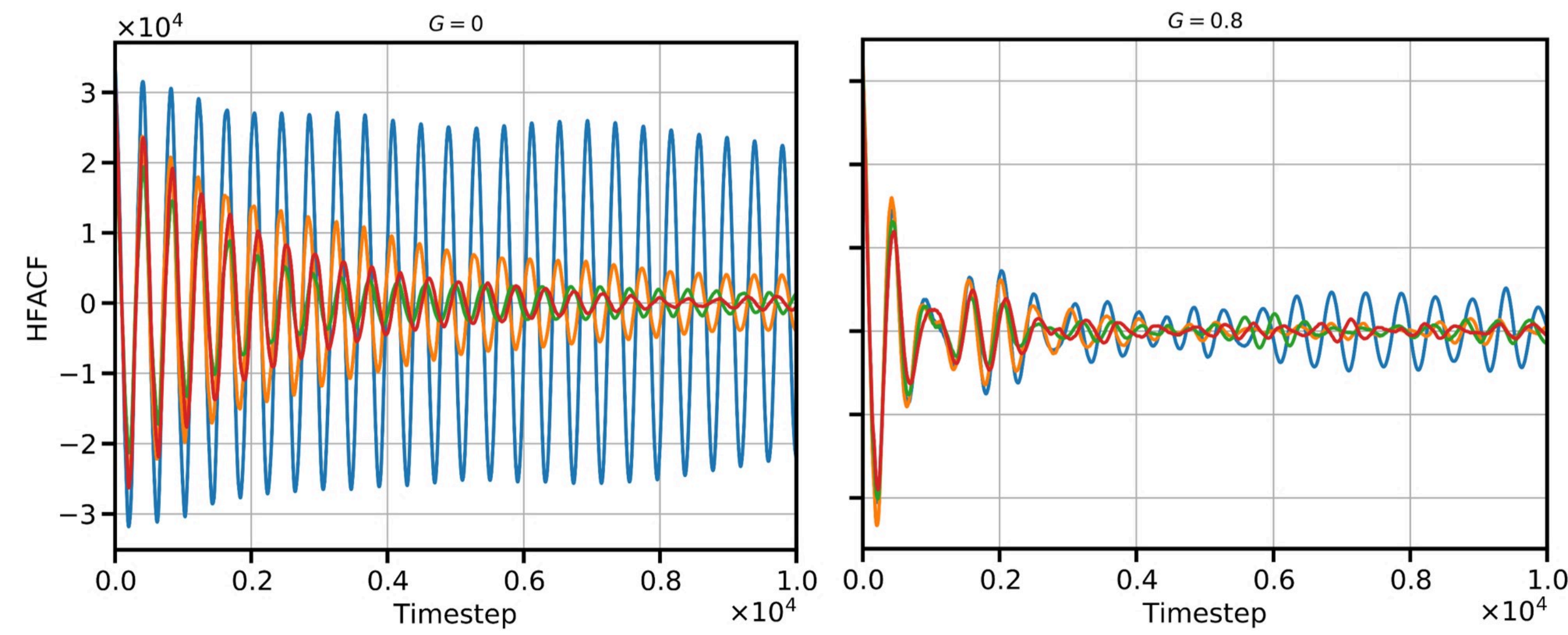

Free Surface $\longrightarrow \varepsilon_{w f}=0.002 \mathrm{eV}$

$\longrightarrow \varepsilon_{w f}=0.04 \mathrm{eV} \quad-\varepsilon_{w f}=0.08 \mathrm{eV}$ 

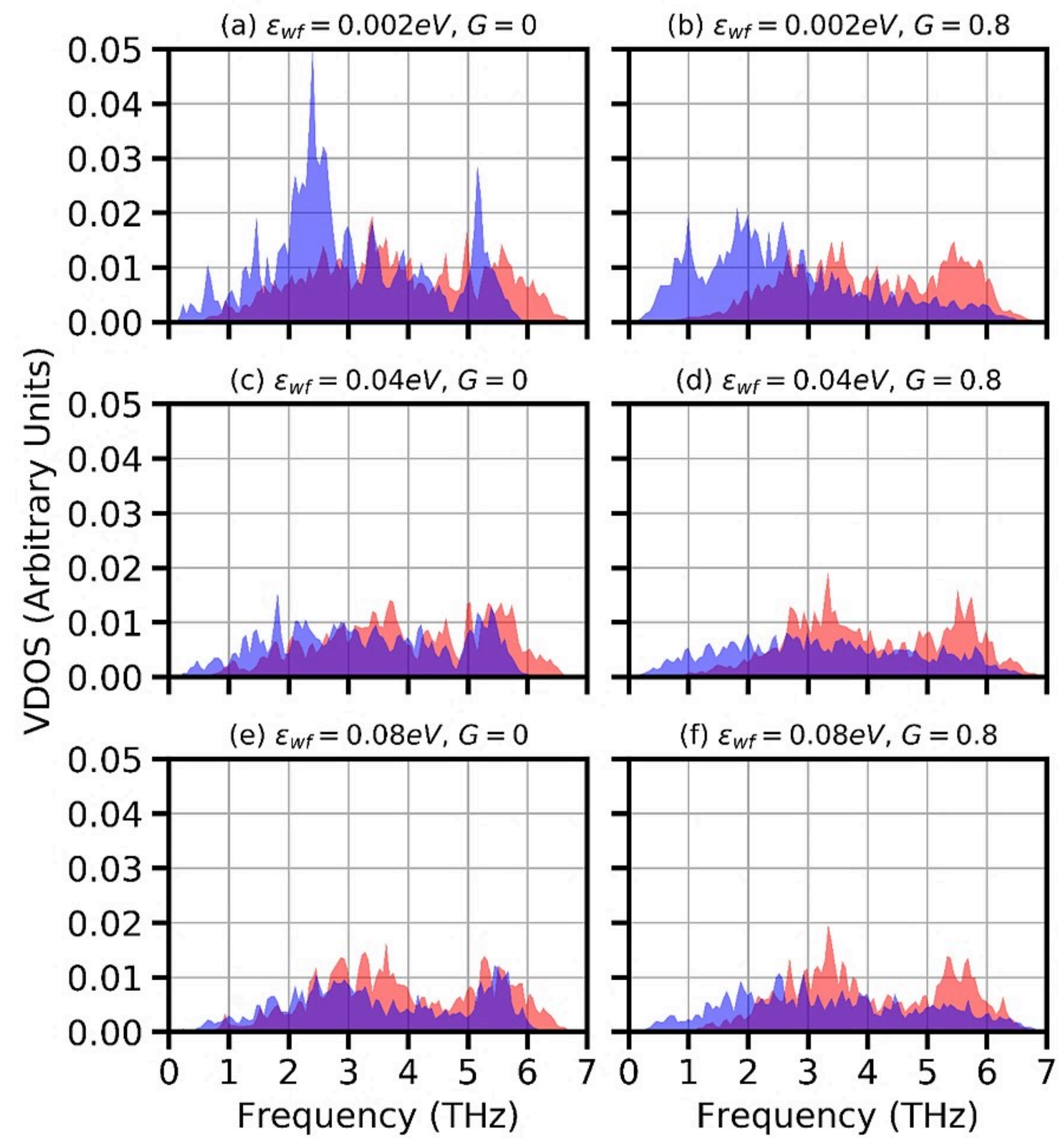

Center Interface Overlap 


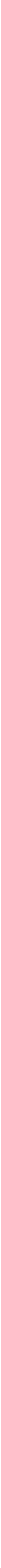




\title{
Cranfield University
}

\section{CERES https://dspace.lib.cranfield.ac.uk}

2019-10-02

\section{Heat transfer across a fractal surface}

\author{
Frank, Michael
}

American Institute of Physics (AIP)

Frank M, Papanikolaou M, Drikakis D, Salonitis K. (2019) Heat transfer across a fractal surface.

Journal of Chemical Physics, Volume 151, October 2019, Article number 134705

https://doi.org/10.1063/1.5115585

Downloaded from Cranfield Library Services E-Repository 